\title{
ECOANATOMÍA FOLIAR DE ARBUSTOS Y ÁRBOLES DEL DISTRITO CHAQUEÑO ORIENTAL DE LA ARGENTINA
}

\author{
ANA ARAMBARRI ${ }^{1,2}$, CLAUDIA MONTI $^{1}$, NÉSTOR BAYÓN¹, MARCELO HERNÁNDEZ¹ ${ }^{1}$ MARÍA CECILIA NOVOA \& \\ MARTA COLARES 1
}

\begin{abstract}
Resumen: Arambarri, A., C. Monti, N. Bayón, M. Hernández, M. C. Novoa \& M. Colares. 2012. Ecoanatomía foliar de arbustos y árboles del distrito chaqueño oriental de la Argentina. Bonplandia 21(1): 5-26.

El objetivo de este trabajo fue evaluar caracteres anatómicos foliares que contribuyan a una mejor comprensión de la relación planta-ambiente y al desarrollo de tecnologías para el aprovechamiento sustentable y conservación de los bosques de esta región. Para ello se aplicaron técnicas histológicas convencionales sobre hojas de ejemplares de 36 especies recolectadas en la región chaqueña húmeda. Se determinaron densidades por $\mathrm{mm}^{-2}$ de células epidérmicas, estomas y tricomas, tipos de mesofilo, tipo y distribución de los tejidos vascular y de sostén. La mayoría de los árboles de la región en estudio presentan hojas hipostomáticas con mesofilo dorsiventral, elevada densidad de células epidérmicas $\left(4000-7000 / \mathrm{mm}^{-2}\right)$, una densidad intermedia de estomas $\left(300-500 / \mathrm{mm}^{-2}\right)$ y baja densidad de tricomas $\left(<35 / \mathrm{mm}^{-2}\right)$. La comparación con los caracteres anatómicos foliares de los arbustos y árboles de las Provincias Biogeográficas Paranaense, de las Yungas y Chaqueña permite concluir que los rasgos más variables son la distribución de estomas y tipo de mesofilo. Las hojas xeromórficas son anfistomáticas, equifaciales y las mesomórficas son hipostomáticas, bifaciales. En el Distrito Chaqueño Oriental las hojas son predominantemente meso-xeromórficas, ya que a sus rasgos mesomórficos se suman otros xeromórficos como elevada densidad de células epidérmicas y abundante tejido esclerenquimático.
\end{abstract}

Palabras clave: Anatomía foliar, Argentina, conservación, plantas nativas forestales, región chaqueña húmeda.

Summary: Arambarri, A., C. Monti, N. Bayón, M. Hernández, M. C. Novoa \& M. Colares. 2012. Leaf ecoanatomy of shrubs and trees from Argentinian Chacoan Oriental District. Bonplandia 21(1): 5-26.

The aim of this paper is to evaluate leaf anatomical traits that contribute to better understanding of the plant-environment relationships, and to the development of technologies for a sustainable use and conservation of Chacoan forests. Conventional histological techniques were applied to leaves of specimens of 36 species collected in the humid Chacoan region. The density of epidermal cells, stomata and trichomes was determined by $\mathrm{mm}^{-2}$. Mesophyll types, and the type and distribution of vascular and esclerenchymatic tissues were also analyzed. Most trees of Chacoan Oriental District presented hypostomatic leaves with dorsiventral mesophyll, high density of epidermal cells $\left(4000-7000 / \mathrm{mm}^{-2}\right)$, intermediate density of stomata $\left(300-500 / \mathrm{mm}^{-2}\right)$,

${ }^{1}$ Docentes-Investigadores del Área de Botánica, Facultad de Ciencias Agrarias y Forestales, UNLP, 60 y 119, CC 31, 1900 La Plata.

22E-mail: anaramba@yahoo.com.ar; botgral@agro.unlp.edu.ar 
and low density of trichomes $\left(<35 / \mathrm{mm}^{-2}\right)$. By comparison leaf anatomical characteristics of shrubs and trees among Paranaense, Yungas and Chacoan Biogeographic Provinces we conclude that the biggest differences can be found within the distribution of stomata and the mesophyll types. Whereas xeromorphic leaves are amphistomatic and equifacial, the mesomorphic are hypostomatic and bifacial. In Chacoan Oriental District, leaves are predominantly mesoxeromorphic, because to their mesomorphic traits added xeromorphic traits such as high density of epidermal cells and abundant sclerenchymatic tissue.

Key words: Argentina, humid Chacoan region, conservation, leaf anatomy, native forest plants.

\section{Introducción}

La Provincia Biogeográfica Chaqueña de la Argentina se divide en cuatro Distritos: Chaqueño Oriental, Chaqueño Occidental, Chaqueño Serrano y el de las Sabanas (Cabrera, 1994). En el presente trabajo nos ocupamos de examinar la anatomía foliar de 36 arbustos y árboles que habitan el Distrito Chaqueño Oriental, el cual ocupa la mitad E de las provincias de Formosa y Chaco, parte del N de Santa Fe y el NO de Corrientes (Fig. 1). El clima es cálido y las precipitaciones varían de 800 a $1.400 \mathrm{~mm}$ anuales, las que aumentan de $\mathrm{O}$ a $\mathrm{E}$ concentrándose en el período estival. La vegetación está formada por bosques xerófilos que se mezclan con palmares, sabanas y selvas marginales a orilla de los ríos, con especies en común con la Provincia Paranaense. Como resultado de la explotación ganadera y forestal, al igual que en el resto de la Provincia Chaqueña, el bosque de este Distrito ha sufrido un deterioro en su estructura y en su composición florística, viéndose afectados principalmente los estratos arbóreo y herbáceo, con la consecuente predominancia de arbustos. Para crear nuevas superficies agrícolas se han quemado o talado los bosques destruyendo gran parte de las especies arbóreas más valiosas (Cabrera, 1994). Estas especies brindan madera empleada en mueblería, fabricación de herramientas, leña, colorantes y principios medicinales (Burkart, 1952, 1979; Digilio \& Legname, 1966; Toursarkissian, 1980; Martínez Crovetto, 1964, 1967, 1981; Legname, 1982; Biloni, 1990; Muñoz, 2000; Arenas, 2003; Carrizo \& al., 2005; Demaio \& al., 2002; Keller \& Romero, 2006; PeñaChocarro \& al., 2006; Cristobal, 2007; Perea \& al., 2007; Juárez de Varela \& Novara, 2007; Keller \& al., 2009; Monte Luchiari da Silva
\& al., 2009; Keller, 2010; Michelin \& al., 2010; Schneider \& al., 2010; Hurrell \& al., 2011). Los estudios botánicos han brindado y brindan conocimientos básicos que pueden constituir herramientas útiles en el diseño de estrategias tendientes a la conservación y manejo sustentable. Sobre la base de lo expuesto, se plantea el objetivo de analizar las características anatómicas de las hojas de 36 especies de arbustos y árboles del Distrito Chaqueño Oriental, con el fin de establecer los caracteres estructurales, en especial aquellos referidos al tejido epidérmico, mesofilo y tejido de sostén que muestren notables variaciones relacionadas con la heterogeneidad ambiental.

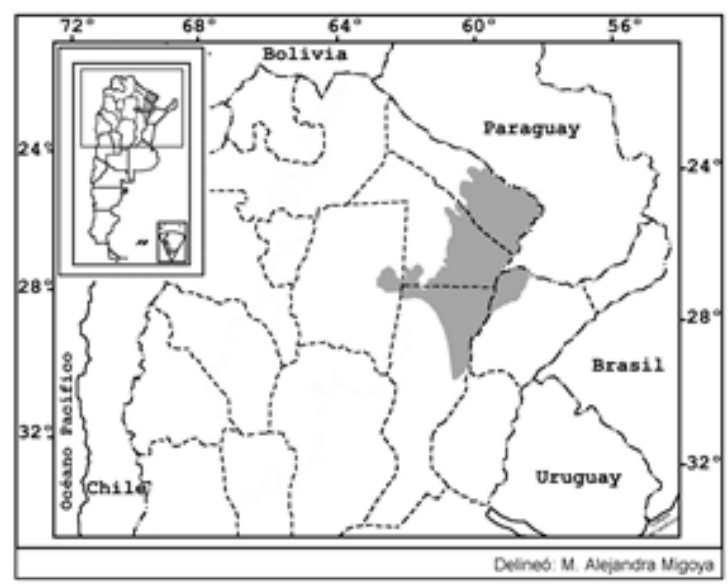

Fig. 1. Mapa del Distrito Chaqueño Oriental (región chaqueña húmeda) de la Argentina.

\section{Material y Métodos}

Se utilizaron especímenes recolectados en el territorio del Distrito Chaqueño Oriental. Estos ejemplares forman parte de las colecciones 
existentes en los herbarios BA, LP, LPAG y SI. En el Apéndice 1 se listan las especies estudiadas, con su nombre científico, familia y los materiales testigos indicando localidades y coleccionistas.

Las hojas de los ejemplares de herbario fueron reconstituidas por imbibición en agua con una gota de detergente en estufa a $30-35^{\circ} \mathrm{C}$ durante 24-72 h, antes de fijarlas en FAA. Para la obtención de las hojas diafanizadas se empleó la técnica de Dizeo de Strittmatter (1973); o bien, se colocó el material en una mezcla de hidróxido de sodio al 5\% e hipoclorito de sodio al $5 \%$, en partes iguales durante 4-5 días. Luego, el material fue lavado con agua destilada y decolorado con hipoclorito de sodio al $50 \%$, nuevamente lavado y colocado por $24 \mathrm{~h}$ en hidrato de cloral al 5\% para su clarificación. Los cortes transversales se realizaron a mano alzada en la parte media de la lámina foliar y en la mitad de la longitud del pecíolo. Los cortes seleccionados fueron decolorados con hipoclorito de sodio al $50 \%$ y lavados con agua caliente para eliminar las burbujas de aire. Tanto los materiales diafanizados como las secciones transversales a mano alzada, sin colorear o coloreados con solución alcohólica de safranina $\mathrm{O}$ al $80 \%$ o violeta de cresilo al $0,5 \%$, fueron montados en gelatina-glicerina (D'Ambrogio de Argüeso, 1986). Asimismo, para realizar los recuentos, las hojas transparentes se montaron temporariamente en glicerina al 90\%. Se contaron células epidérmicas, estomas y tricomas en ocho campos, ubicados en la parte media de los semilimbos de la lámina y sobre ambas epidermis. Las células y estomas que se encontraban en el borde del campo visual no fueron considerados en el recuento. Esta metodología se repitió en dos a cuatro muestras por especie. La densidad promedio de los tipos celulares se expresó por milímetro cuadrado $\mathrm{mm}^{-2}$ (Tabla 1). Para las células epidérmicas se consideraron los siguientes rangos de densidad: baja $<3000$; intermedia 3000-4000; alta 40007000; muy alta $>7000$ células epidérmicas/ $\mathrm{mm}^{-2}$. Para los estomas fueron: baja $<300$; intermedia 300-500; alta 500-1000; muy alta $>1000$ estomas/ $\mathrm{mm}^{-2}$. Por último, para los tricomas se consideraron los siguientes rangos: ausentes 0 ; baja $<35$; intermedia $35-70$ y alta $>70$ tricomas $/ \mathrm{mm}^{-2}$. Para el cálculo del índice estomático se aplicó la fórmula de Salisbury
(1929): [nro. de estomas / (nro. de estomas + nro. células epidérmicas)] x 100.

Las descripciones anatómicas y morfológicas fueron realizadas según la terminologíautilizada por Metcalfe \& Chalk (1950, 1979, 1989), Stace (1965) y Bianco \& al. (2004). Para describir las características ecoanatómicas foliares (Tabla 2) se consideró a las hojas tipo sol (xeromorfismo), tipo intermedia (mesomorfismo) y tipo sombra (hidromorfismo), según sus caracteres representativos y propios para cada condición (Roth, 1984).

En el análisis de los preparados se utilizó un microscopio óptico Leitz SM lux. Para las observaciones, captura y digitalización de las imágenes se empleó un microscopio Gemalux equipado con una cámara color PAL CCD conectada al software Hyper Media Center.

Para el estudio de los rasgos topográficos con microscopio electrónico de barrido (MEB), las hojas de un grupo selecto de especies se deshidrataron con una serie alcohólica ascendente siguiendo el método del silicagel (D’Ambrogio de Argüeso, 1986). Porciones de 2-5 milímetros se adhirieron a los soportes y metalizaron con oro-paladio. Se observaron y tomaron fotografías con el microscopio electrónico de barrido y microanálisis del Centro de Investigación y Desarrollo en Ciencias Aplicadas CINDECA (CONICET/ UNLP). Para la nomenclatura de las especies se consultó el Catálogo de Cono Sur (Zuloaga \& al., 2008) y las bases de datos del Instituto de Botánica Darwinion (www.darwin.edu.ar/ en mayo 2010) y del Missouri Botanical Garden (http://www.tropicos.org en julio de 2011).

\section{Resultados y Discusión}

\section{Células epidérmicas}

Vista en superficie las paredes anticlinales de las células epidérmicas de la cara adaxial son rectas a levemente curvadas en 26 de las 36 especies estudiadas (e.g. Aspidosperma quebracho-blanco, Erythrina dominguezii, Genipa americana, Geoffroea decorticans, Peltophorum dubium, Prosopis affinis, $P$. alba, P. nigra, Sapindus saponaria) (Figs. $2 \mathrm{~A}$ y $4 \mathrm{~A}, \mathrm{~B})$, y curvado-onduladas hasta sinuosas en las especies restantes (Fig. 2B- 
E). Más del $70 \%$ de las especies presentaron epidermis con características xeromórficas, en ambas caras de la hoja; las células son de paredes anticlinales rectas o solo levemente curvadas. La ornamentación cuticular y/o espesor de la cutícula fue notable en más del $60 \%$ de las especies: Allophylus edulis, Aspidosperma quebracho-blanco, Astronium balansae, Casearia sylvestris, Chrysophyllum marginatum, Enterolobium contortisiliquum, Guarea macrophylla subsp. spicaeflora, Guazuma ulmifolia var. ulmifolia, Handroanthus heptaphyllus, Holocalyx balansae, Maytenus ilicifolia, Patagonula americana, Peltophorum dubium, Pouteria salicifolia, Prosopis spp., Pterogynenitens, Salix humboldtiana, Sapindus saponaria, Tabebuia aurea, Tabernaemontana catharinensis y Trichilia catigua (Figs. 2E y 3A).

El depósito de ceras epicuticulares fue evidente al microscopio óptico, en la epidermis de 18 de las 36 especies: Allophylus edulis, Anadenanthera colubrina var. cebil, Caesalpinia paraguariensis (Fig. 2F), Casearia sylvestris, Enterolobium contortisiliquum, Eugenia uniflora, Genipa americana, Guarea macrophylla subsp. spicaeflora, Handroanthus heptaphyllus, Maytenus ilicifolia, Peltophorum dubium, Pouteria gardneriana, Prosopis spp. (Fig. 4A), Pterogyne nitens y Rollinia emarginata. El depósito de ceras constituye una efectiva protección contra la desecación siendo una característica xeromórfica o de plantas expuestas a intensa radiación solar o sequía (Roth, 1984; Arambarri \& al., 2011).

Las células epidérmicas en corte transversal frecuentemente muestran una pared periclinal externa gruesa y cubierta por capas de ceras. La cutícula es delgada de 1-3 $\mu \mathrm{m}$ de espesor, gruesa 4-5 $\mu \mathrm{m}$ (e.g. Peltophorum dubium y Trichilia catigua), hasta muy gruesa en Aspidosperma quebracho-blanco con 10 $\mu \mathrm{m}$ de espesor. Esta última especie presenta una epidermis unistrata formada por células alargadas en sentido perpendicular a la superficie de la hoja (Fig. 3A). En general, las epidermis son unistratas; sin embargo, algunas especies presentan epidermis pluristrata o una hipodermis, como ocurre en Geoffroea decorticans, Guazuma ulmifolia var. ulmifolia, Handroanthus heptaphyllus, Myrcianthes
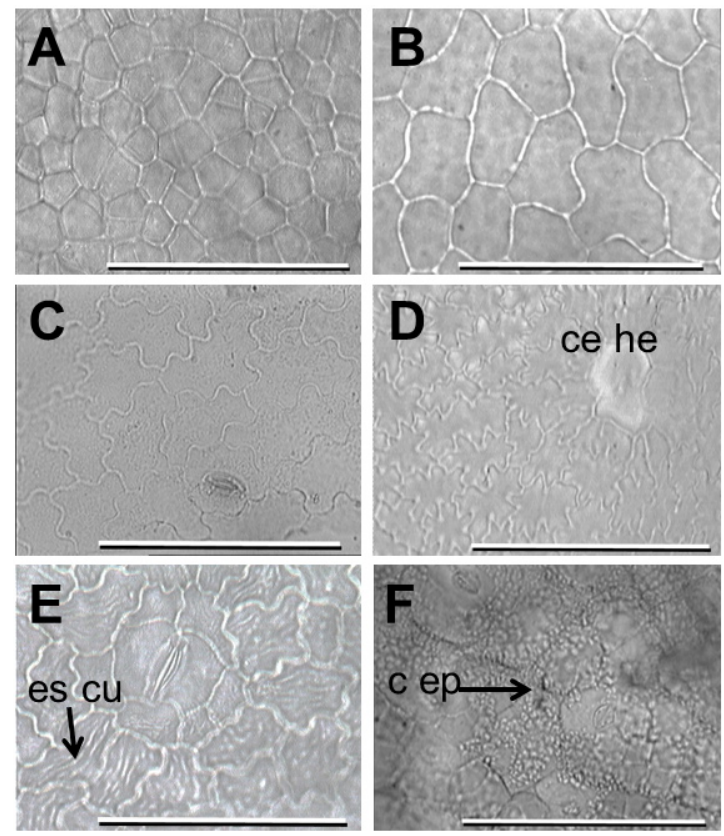

Fig. 2. Epidermis vista en superficie. A: Peltophorum dubium, epidermis adaxial, paredes anticlinales rectas. B: Astronium balansae, epidermis adaxial, paredes anticlinales curvadas. C: Caesalpinia paraguariensis, epidermis adaxial, paredes anticlinales sinuosas en U. D: Myrcianthes pungens, epidermis adaxial, paredes anticlinales ondulado-sinuosas en forma de $\mathrm{V}$ y un par de células heteromorfas (ce he). E: Guarea macrophylla subsp. spicaeflora, mostrando estrías cuticulares (es cu). F: Caesalpinia paraguariensis, epidermis cubierta por abundantes ceras epicuticulares (c ep), con aspecto de gránulos brillantes. Escalas: $100 \mu \mathrm{m}$.

pungens, Peltophorum dubium y Tabebuia aurea (Fig. 3B, C). Las epidermis pluristratas halladas en Handroanthus heptaphyllus y Tabebuia aurea, coinciden con las descripciones realizadas por Silva \& al. (2009) para las especies de ambos géneros. La presencia de epidermis bi-pluristrata, o unistrata con células mucilaginíferas, o la epidermis acompañada por una hipodermis son adaptaciones xeromórficas, halomórficas o a condiciones rigurosas, ya que tienen por función economizar agua y también actuar en la protección del clorénquima de la excesiva radiación solar (Roth, 1984, 1990, 1995), y cuando sus paredes están engrosadas (e.g. hipodermis de Myrcianthes pungens) contribuyen a evitar el colapso celular reforzando la estructura foliar (Fahn \& Cutler, 1992; Cosa \& Dottori, 2010). 
Algunas especies tienen epidermis papilosa (Fig. 3D). Las papilas se visualizan como proyecciones cónicas de la pared periclinal externa. Se observan en ambas epidermis de Caesalpinia paraguariensis y Enterolobium contortisiliquum y solo en la epidermis abaxial de Anadenanthera colubrina var. cebil, Carica quercifolia, Pouteria gardneriana y Terminalia triflora. De acuerdo a lo referido por Metcalfe \& Chalk (1979), las papilas se encuentran preferentemente en la epidermis abaxial de una amplia variedad de plantas, con diferentes portes y hábitats, pero son frecuentes en las que habitan bajo condiciones rigurosas. $\mathrm{Su}$ presencia en la vegetación xerófita de la Puna de Mendoza fue comunicada por Ancibor (1992). También se encontraron en especies de los Distritos Chaqueños Occidental y Serrano, con condiciones climáticas semiáridas y alta luminosidad (Arambarri \& al., 2011). Este carácter frecuente en algunas familias como las
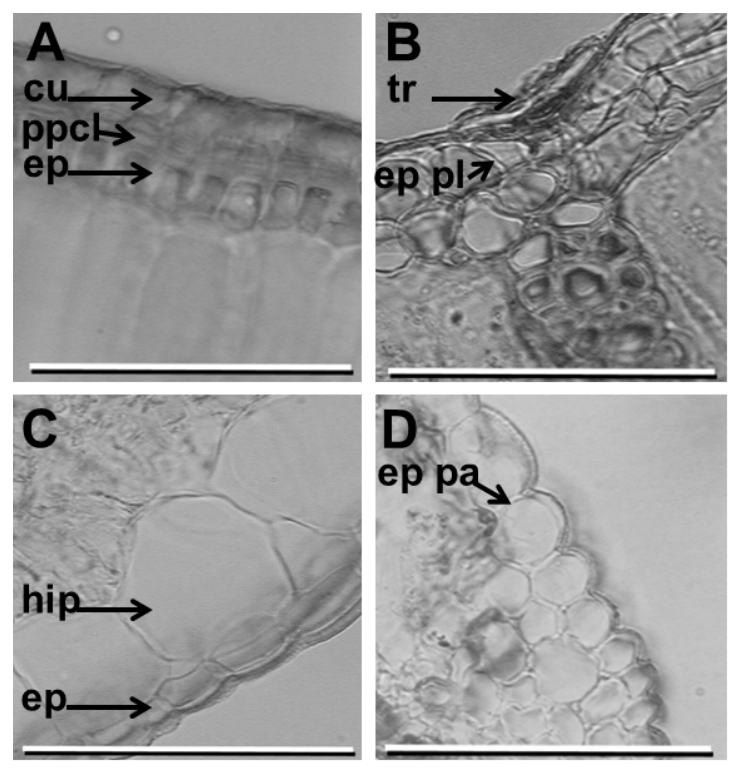

Fig. 3. Epidermis en corte transversal. A: Aspidosperma quebracho-blanco, epidermis unistrata (ep) con células epidérmicas alargadas en sentido perpendicular al órgano, mostrando una pared periclinal externa engrosada (ppcl) y cutícula (cu) de $10 \mu \mathrm{m}$ de espesor. B: Tabebuia aurea, epidermis pluristrata (ep pl) y un tricoma escamoso peltado (tr) con el pie hundido en la epidermis. C: Geoffroea decorticans, epidermis abaxial unistrata (ep) e hipodermis (hip). D: Caesalpinia paraguariensis, epidermis unistrata papilosa (ep pa). Escalas: $100 \mu \mathrm{m}$.
Fabaceae podría tener algún valor taxonómico (Roth, 1984). Se presume que la formación de papilas responde a factores genéticos y se ve afectada por los factores climáticos. Por un lado, se les ha atribuido la función de concentrar los rayos de luz en plantas que crecen en el sotobosque (Haberlandt, 1914), lo que podría justificar la existencia de epidermis papilosas en la vegetación arbustiva de las Yungas (Arambarri, \& al., 2009a, 2009b). Por otro lado, se ha observado que en las epidermis papilosas los estomas quedan hundidos entre las papilas, lo cual les brinda protección en plantas de ambientes xéricos (Fahn \& Cutler, 1992).

La densidad de células epidérmicas frecuentemente es elevada. El 50\% de las especies tiene entre 4000 y 7000 células epidérmicas por milímetro cuadrado, e. g. Prosopis alba (Fig. 4A). Una densidad mayor a 7000 células epidérmicas $/ \mathrm{mm}^{-2}$ se halló en Allophylus edulis, Enterolobium contortisiliquum, Peltophorum dubium (Fig. 2A), Sapindus saponaria (Fig. 4B) y Tabebuia aurea. Por último, Aspidosperma quebrachoblanco mostró en ambas epidermis más de 10000 células epidérmicas $/ \mathrm{mm}^{-2}$ (Tabla 1$)$. Los mismos resultados se hallaron en las especies Bulnesia sarmientoi Lorentz ex Griseb., Schinopsis balansae Engl., S. lorentzii (Griseb.) Engl. y Scutia buxifolia Reissek, de los distritos semiáridos chaqueños (Arambarri \& al., 2011). La elevada densidad de células epidérmicas se corresponde con la reducción del tamaño y volumen de las células, a veces acompañada de un incremento del espesor de las paredes (Figs. 2E y 3A), que reducen las posibilidades de colapso celular por deshidratación (Fahn \& Cutler, 1992; Cosa \& Dottori, 2010).

\section{Estomas}

El tipo de estomas anomocítico se encuentra en más del $70 \%$ de las especies estudiadas. Como único tipo de estoma se observa en Allophylus edulis, Aspidosperma quebrachoblanco, Carica quercifolia, Guazuma ulmifolia var. ulmifolia, Handroanthus heptaphyllus, Holocalyx balansae, Myrcianthes pungens, Patagonula americana, Peltophorum dubium, Pterogyne nitens, Tabebuia aurea y Terminalia triflora. Los estomas paracíticos se observan 
en: Anadenanthera colubrina var. cebil, Casearia sylvestris, Erythrina dominguezii, Genipa americana, Ocotea diospyrifolia, Prosopis affinis, P. alba (Fig. 4A), P. nigra, Rollinia emarginata y Salix humboldtiana. Los resultados coinciden con la cita previa de este tipo de estoma como característico de las familias Annonaceae, Lauraceae, Rubiaceae y Salicaceae (Arambarri \& al., 2006, 2008, 2009a y 2009b). Se concluye que estomas de tipo anomocítico y paracítico (en menor medida) son los que predominan en los arbustos y árboles, resultando coincidente con lo referido por Cronquist (1988) sobre que el hábito arbóreo de las plantas como estos tipos de estomas son caracteres primitivos.

Un grupo de especies presenta más de un tipo de estomas, por ejemplo: a) anomocíticos y paracíticos en Caesalpinia paraguariensis, Eugenia uniflora, Guarea macrophylla subsp. spicaeflora y Trichilia catigua; b) anomocíticos y anisocíticos en Chrysophyllum marginatum, Enterolobium contortisiliquum y Grabowskia duplicata; c) anomocíticos, anisocíticos $\mathrm{y}$ paracíticos en Geoffroea decorticans y Prosopis kuntzei; d) anomocíticos y ciclocíticos en Maytenus ilicifolia y Pouteria gardneriana; e) anomocíticos y actinocíticos en Astronium balansae y Sapindus saponaria (Fig. 4B). La presencia de varios tipos de estomas en una misma epidermis es un hecho constatado por numerosos autores (e.g. Bernardello, 1982; Cosa de Gastiazoro, 1991; Lorca \& al., 1995; Cortadi \& al., 1996; Colares \& al., 1999; Stenglein \& al., 2003; Guantay 2004; Licovsky \& Cosa, 2005; Freire \& al., 2005; Rigonatto \& al., 2005). Una notable adaptación xeromórfica la constituyen los estomas con anillo peristomático que se observan en Aspidosperma quebracho-blanco (Fig. 5A, B); carácter xeromórfico que se suma
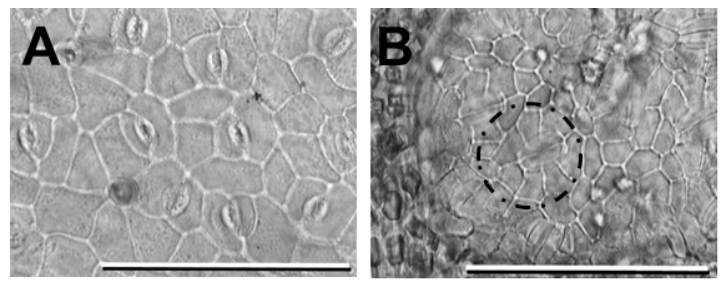

Fig. 4. Estomas. A: Prosopis alba, paracíticos. B: Sapindus saponaria, actinocítico indicado con línea cortada. Escalas: $100 \mu \mathrm{m}$. a los ya citados para las células epidérmicas de la misma especie.

En lo referente a la distribución de estomas en la lámina foliar, el 70\% de las especies mostraron hojas hipostomáticas. Mientras que, resultaron anfistomáticas las hojas de Anadenanthera colubrina var. cebil, Aspidosperma quebrachoblanco, Caesalpinia paraguariensis, Erythrina dominguezii, Geoffroea decorticans, Prosopis affinis, P. alba, P. kuntzei, P. nigra, Salix humboldtiana y Tabebuia aurea. Todas ellas de ambientes xéricos o bien sus copas, en altura, se caracterizan por quedar expuestas a una intensa luminosidad desarrollando caracteres xeromórficos. Los resultados coinciden con Fahn \& Cutler (1992), quienes señalan que las hojas de plantas de ambientes xéricos son frecuentemente anfistomáticas, ya que uno de los factores que induce la formación de estomas en la superficie adaxial es la exposición a una intensa luminosidad.

Dentro del rango de densidad estomática que se estableció para el análisis de las especies, la mayoría mostró en la cara abaxial una densidad intermedia entre 300-500 estomas/ $\mathrm{mm}^{-2}$ (e.g. Astronium balansae, Caesalpinia paraguariensis, Erythrina dominguezii, Genipa americana, Geoffroea decorticans, Handroanthus heptaphyllus, Holocalyx balansae, Prosopis alba, Rollinia emarginata, Tabebuia aurea). Algunas especies mostraron, en la cara abaxial, una densidad estomática alta (500-900 estomas $\left./ \mathrm{mm}^{-2}\right)$, Enterolobium contortisiliquum, Eugenia uniflora, Patagonula americana, Peltophorum dubium, Prosopis kuntzei, Pterogyne nitens y Sapindus saponaria, de las cuales, excepto Prosopis kuntzei las restantes son hipostomáticas. Tres especies
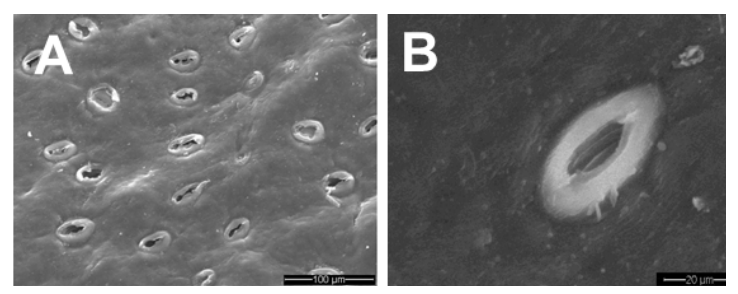

Fig. 5. Fotomicrografías con MEB. Aspidosperma quebracho-blanco: A: vista general de la cubierta de ceras lisa y estomas distribuídos al azar. B: Detalle de un estoma mostrando el anillo peristomático. Escalas: A: $100 \mu \mathrm{m}$. B: $20 \mu \mathrm{m}$. 
alcanzaron en la cara abaxial, valores muy altos de densidad estomática (mayor a 1000 estomas $/ \mathrm{mm}^{-2}$ ), Anadenanthera colubrina var. cebil, Casearia sylvestris y Guazuma ulmifolia var. ulmifolia (Tabla 1). De acuerdo con Roth (1984) las plantas que viven en ambientes xéricos desarrollan un elevado número de estomas de pequeñas dimensiones, los cuales les permite regular mejor la pérdida de agua comparado con un bajo número de estomas grandes. Los resultados también concuerdan con Fahn \& Cutler (1992), quienes señalan que las plantas de regiones xéricas presentan una mayor densidad de estomas que las que viven en regiones mésicas.

Las plantas generalmente presentan mayor densidad de estomas en la cara abaxial que en la adaxial; sin embargo, en Geoffroea decorticans, Prosopis affinis, P. alba, P. kuntzei y P. nigra la mayor densidad de estomas se encuentra en la superficie adaxial. Igual registro fue hallado para especies de los géneros Acacia y Cercidium por Arambarri \& al. (2011). De acuerdo con Fahn \& Cutler (1992), la intensidad lumínica induce la formación de estomas en la superficie adaxial de la hoja, y como consecuencia de ello se reduce el número de estomas en la superficie abaxial. Se destaca que Aspidosperma quebrachoblanco, Carica quercifolia, Chrysophyllum marginatum, Grabowskia duplicata y Salix humboldtiana presentaron densidad estomática menor a 300 estomas $/ \mathrm{mm}^{-2}$. La reducción de la densidad de estomas es consecuencia de diferentes adaptaciones. Fahn \& Cutler (1992) refieren que las hojas suculentas reducen la densidad estomática. Bianco \& al. (2004) y Cosa \& Dottori (2010), mencionan que las halófitas presentan hojas crasas y baja densidad estomática. Estas últimas características están presentes en Grabowskia duplicata (Tablas 1 y 2).

\section{Índice estomático}

El índice estomático más frecuente es inferior al $10 \%$ en Aspidosperma quebracho-blanco, Astronium balansae, Carica quercifolia, Chrysophyllum marginatum, Enterolobium contortisiliquum (en la muestra de Chaco), Geoffroea decorticans, Grabowskia duplicata, Guarea macrophylla subsp. spicaeflora, Handroanthus heptaphyllus, Holocalyx balansae, Maytenus ilicifolia (en la muestra de Chaco), Ocotea diospyrifolia, Patagonula americana, Peltophorum dubium, Pouteria gardneriana, Prosopis affinis, P. alba, P. kutzei, P. nigra (en el género Prosopis solo en la cara abaxial), Pterogyne nitens, Salix humboldtiana, Sapindus saponaria, Tabebuia aurea, Tabernaemontana catharinensis (en la muestra de Chaco) y Trichilia catigua (Tabla 1). Se aclara que en algunas especies, el índice estomático tuvo un valor menor en ejemplares recolectados en la Provincia Chaqueña que en las Provincias Paranaense y de las Yungas. Es decir, que las condiciones de baja humedad y alta luminosidad reducirían el índice estomático. Estos resultados coinciden con los referidos por Arambarri \& al. (2009a y 2009b), al estudiar el índice estomático de arbustos y árboles de la Provincia de las Yungas, donde concluyeron que el valor se reducía al aumentar el porte de los árboles, relacionando un valor de índice estomático inferior al $10 \%$ con condiciones de alta luminosidad, acción de vientos deshidratantes y/o sequías temporarias que provocan en el follaje de estos árboles el desarrollo de características xeromórficas similares a las de zonas semiáridas.

\section{Tricomas}

El 83\% de las especies presentan tricomas glandulares y/o eglandulares, con estructura celular constante y de valor taxonómico. Siguiendo a Metcalfe \& Chalk (1979), se destacan los tricomas escamoso-peltados en depresiones de la epidermis en Handroanthus heptaphyllus y Tabebuia aurea (Figs. 3B y 6A, B).
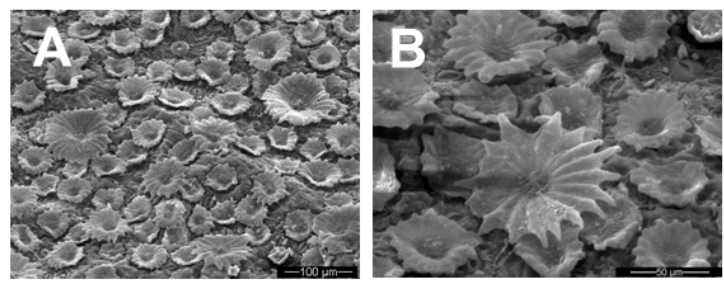

Fig. 6. Fotomicrografîas con MEB. A: Tabebuia aurea, epidermis cubierta por tricomas escamosos peltados. B: Detalle de los tricomas, donde se muestran las células de la escama con disposición radiada respecto al centro, donde se inserta en el pie. Escalas: A: $100 \mu \mathrm{m}$. B: 50 $\mu \mathrm{m}$. 
Los tricomas malpighiáceos o en forma de $\mathrm{T}$, en Pouteria gardneriana y tricomas combretáceos, bicelulares con la célula basal transparente, en Terminalia triflora ya fueron mencionados por Leonardi \& al. (2002) y Arambarri \& al. (2006, 2009a, 2009b). En las especies de Prosopis se hallaron tricomas eglandulares con pared gruesa y rugosa (Fig. 7A). Mientras que, en Geoffroea decorticans, Holocalyx balansae y Peltophorum dubium se encontró un tipo particular de tricoma simple, uniseriado, bicelular con la célula basal completamente cutinizada, similar al descripto por Fahn \& Cutler (1992). Los mismos autores explican que la célula basal con esas características es una adaptación al ambiente xérico, ya que impide la penetración apoplástica del agua en el tricoma y de esa manera se evita la evaporación de agua a través de las paredes de los tricomas muertos. En Astronium balansae, se halló el tricoma glandular característico de la familia Anacardiaceae (Arambarri \& al., 2006, 2008, 2009a), ilustrado por Ruiz \& al. (2009). En las hojas juveniles de Caesalpinia paraguariensis se observó un tricoma glandular formado por un pie multicelular y una cabeza globosa también multicelular (Fig. 7B-D).
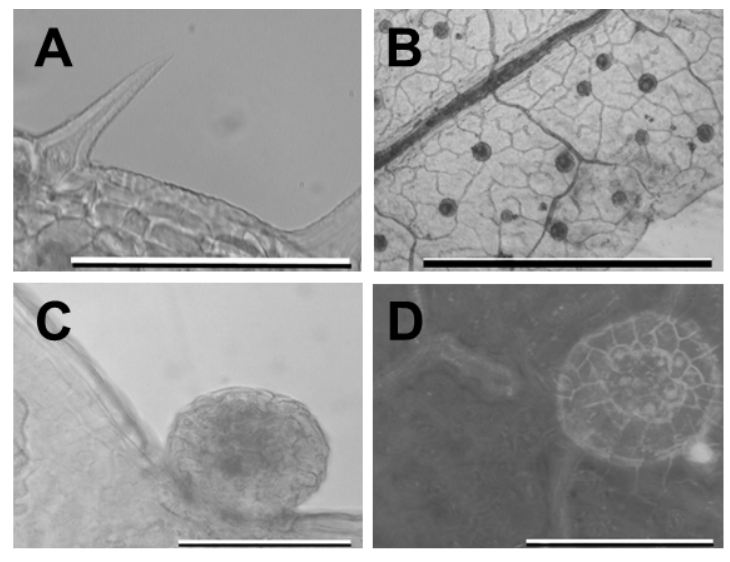

Fig. 7. Tricomas. A: Prosopis spp., eglandular, unicelular, agudo, con la superficie rugosa. B, C, D: Caesalpinia paraguariensis. B: Vista en superficie de la lámina del foliólulo juvenil mostrando los tricomas glandulares distribuidos en la superficie y margen. C: Detalle de un tricoma glandular en el margen del foliólulo. D: Detalle del mismo tricoma visto en superficie donde se distingue el pie y la cabeza, ambos multicelulares. Escalas: A, C, D: $100 \mu \mathrm{m}$. B: $1 \mathrm{~mm}$.
La densidad de tricomas es un carácter variable. Una elevada densidad de tricomas/ $\mathrm{mm}^{-2}$ se halló en Aspidosperma quebrachoblanco (muestra de Santiago del Estero), Geoffroea decorticans, Prosopis affinis, P. alba, P. kuntzei, P. nigra y Tabebuia aurea. La mayoría de las especies presentaron una cobertura de la superficie foliar inferior a 35 tricomas $/ \mathrm{mm}^{-2}$ y seis especies fueron glabras: Carica quercifolia, Chrysophyllum marginatum, Eugenia uniflora, Genipa americana, Maytenus ilicifolia y Tabernaemontana catharinensis (Tabla 1). Al comparar los resultados de trabajos de anatomía foliar de los árboles y arbustos de diferentes regiones biogeográficas se observa que el 95\% de las especies de la Provincia de las Yungas presentan tricomas en sus hojas (Arambarri \& al., 2009b); mientras que, en la Provincia Chaqueña esta proporción varía entre un $87 \%$ para el Distrito Oriental (húmedo) y un $72 \%$ para los Distritos Occidental y Serrano (semiáridos). Se observa que el número de especies con indumento en zonas húmedas es mayor que en zonas semiáridas. Se infiere que la presencia de abundantes ceras epicuticulares, un incremento en el espesor de la cutícula y el desarrollo de epidermis bi-pluristrata e hipodermis reemplazan la función de los tricomas como reguladores de la temperatura foliar, absorbancia de radiación solar y consiguiente regulación de la economía del agua, siguiendo la observación referida por Fahn \& Cutler (1992).

\section{Mesofilo}

El 50\% de las especies exhibe mesofilo dorsiventral: Allophylus edulis, Caesalpinia paraguariensis, Carica quercifolia, Casearia sylvestris, Chrysophyllum marginatum, Eugenia uniflora, Genipa americana, Guarea macrophylla subsp. spicaeflora, Guazuma ulmifolia var. ulmifolia, Handroanthus heptaphyllus, Holocalyx balansae, Ocotea diospyrifolia, Patagonula americana, Prosopis kuntzei, Rollinia emarginata, Sapindus saponaria, Tabernaemontana catharinensis y Trichilia catigua (Fig. 8A). El 33\% de las especies presenta mesofilo isolateral o en empalizada (e.g. Anadenanthera colubrina var. cebil, Aspidosperma quebracho-blanco, Erythrina dominguezii, Geoffroea decorticans, 
Grabowskia duplicata, Peltophorum dubium, Pouteria gardneriana, Prosopis affinis, $P$. alba, P. nigra, Tabebuia aurea y Terminalia triflora. En el 17\% de las especies el mesofilo es variable, e.g. Astronium balansae, Enterolobium contortisiliquum, Maytenus ilicifolia, Myrcianthes pungens, Pterogyne nitens y Salix humboldtiana (Tabla 2); estas últimas, serían las especies que poseen mayor plasticidad adaptativa. Un caso especial es Prosopis kuntzei, que siendo una especie de ambiente xérico muestra características epidérmicas y de mesofilo mesomórficas, lo cual se puede atribuir a lo efímero de su follaje.

\section{Vena media en corte transversal}

Las secciones transversales de las venas medias presentan la cara adaxial convexa, plana o cóncava; mientras que, la cara abaxial generalmente es convexa, rara vez plana. De esta manera, de acuerdo a la forma de las caras adaxial y abaxial la sección transversal de la vena media se ha clasificado en biconvexa, plano-convexa, cóncavo-convexa o biplana. En el presente trabajo la vena media es biconvexa en 20 de las 36 especies (Allophylus edulis, Aspidosperma quebracho-blanco, Astronium balansae, Carica quercifolia, Chrysophyllum marginatum, Erythrina dominguezii, Grabowskia duplicata, Guazuma ulmifolia var. ulmifolia, Holocalyx balansae, Maytenus ilicifolia, Ocotea diospyrifolia, Patagonula americana, Pouteria gardneriana, Prosopis affinis, P. nigra, Salix humboldtiana, Sapindus saponaria, Tabebuia aurea, Terminalia triflora y Trichilia catigua). En las especies restantes es plano-convexa o cóncavo-convexa (e.g. Handroanthus heptaphyllus), con excepción de Geoffroea decorticans y Peltophorum dubium que presentan sección biplana. Al comparar estos resultados con los de estudios previos (Arambarri \& al., 2006, 2008, 2009a, 2009b), se concluye que la más frecuente es la sección biconvexa.

\section{Haces vasculares}

El tejido vascular en la vena media se dispone en posición abaxial formando un haz o grupo de haces colaterales unidos en Anadenanthera colubrina var. cebil, Caesalpinia paraguariensis, Casearia sylvestris, Enterolobium contortisiliquum, Geoffroea decorticans, Guazuma ulmifolia var. ulmifolia, Holocalyx balansae, Ocotea diospyrifolia, Peltophorum dubium, Prosopis affinis, P. alba, P. kuntzei, P. nigra, Pterogyne nitens, Rollinia emarginata y Tabebuia aurea. Más frecuentemente se encuentran haces colaterales abaxiales y adaxiales inversos con el xilema interno.

\section{Esclerénquima}

La mayoría de las especies presentan tejido esclerenquimático. Este tejido de sostén característico de las venas está dispuesto en forma de casquetes floemático y xilemático o formando un anillo esclerenquimático perivascular. Este tejido es más abundante en especies xeromórficas que en mesomórficas. En algunas especies, hay además esclereidas en el parénquima de la lámina o del pecíolo (e.g. Guarea macrophylla subsp. spicaeflora, Maytenus ilicifolia y Rollinia emarginata) y en Aspidosperma quebracho-blanco las células del parénquima en empalizada del mesofilo en su mayoría se han transformado en esclereidas idioblásticas. Este carácter se suma a la presencia de un mesofilo isolateral (ambos ilustrados por Cosa \& al., 2011), elevada densidad de células epidérmicas y baja densidad de estomas que parecen indicar la adaptación de esta especie a suelos salinos. Por el contrario, las fibras están ausentes o son escasas en Astronium balansae, Caesalpinia paraguariensis, Caricaquercifolia, Grabowskia duplicata y Tabernaemontana catharinensis.

\section{Cristales}

Más del 50\% de las especies presentan cristales solitarios y formando maclas, Anadenanthera colubrina var. cebil, Caesalpinia paraguariensis, Chrysophyllum marginatum, Enterolobium contortisiliquum, Erythrina dominguezii, Geoffroea decorticans, Guazuma ulmifolia var. ulmifolia, Handroanthus heptaphyllus, Holocalyx balansae, Maytenus ilicifolia, Ocotea diospyrifolia, Pouteria gardneriana, Prosopis spp. (Fig. 8B), Pterogyne nitens, Salix humboldtiana y Tabebuia aurea. Se encuentran drusas en Allophylus edulis, Aspidosperma quebracho-blanco, Astronium balansae, 
Carica quercifolia, Casearia sylvestris, Eugenia uniflora, Genipa americana, Guarea macrophylla subsp. spicaeflora, Myrcianthes pungens, Peltophorum dubium, Rollinia emarginata, Salix humboldtiana, Sapindus saponaria, Tabernaemontana catharinensis, Terminalia triflora y Trichilia catigua. Por último, dos especies (Grabowskia duplicata y Patagonula americana) poseen arena cristalina. Este último resultado coincide con los conceptos vertidos previamente por Arambarri \& al. (2006, 2008, 2009b).
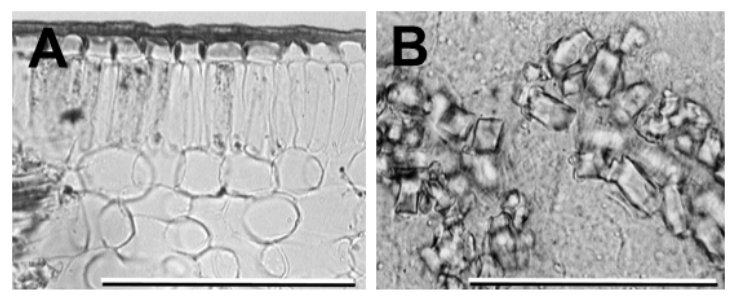

Fig. 8. Mesofilo y cristales. A: Trichilia catigua, mesofilo dorsiventral. B: Prosopis kuntzei, cristales en el mesofilo. Escalas: $100 \mu \mathrm{m}$.

\section{Pecíolo en sección transversal}

El contorno más frecuente es redondeado y con lóbulos, como se observa en Anadenanthera colubrina var. cebil, Erythrina dominguezii, Genipa americana, Geoffroea decorticans, Grabowskia duplicata, Guarea macrophylla subsp. spicaeflora, Handroanthus heptaphyllus, Holocalyx balansae, Maytenus ilicifolia, Patagonula americana, Peltophorum dubium, Pouteria gardneriana, Prosopis affinis, P. alba, P. kuntzei, P. nigra, Pterogyne nitens, Rollinia emarginata, Tabebuia aurea, Terminalia triflora y Trichilia catigua. Los lóbulos son alados en Sapindus saponaria y Tabernaemontana catharinensis. Los pecíolos de las 13 especies restantes no presentan lóbulos. La epidermis es unistrata, generalmente con cutícula gruesa y pubescente. La corteza está formada por colénquima y parénquima interno o parénquima colenquimatoso donde se encuentran cristales, esclereidas idioblásticas y/o tejidos de secreción. El tejido vascular, presenta características que se corresponden con la vena media de la hoja.

\section{Conclusiones}

Los arbustos y árboles del Distrito Chaqueño Oriental se caracterizan por tener hojas mesoxeromórficas. El 69\% de las especies posee hojas mesomórficas, es decir hipostomáticas con mesofilo dorsiventral. A estos caracteres en un $55 \%$ de las especies se le suman algunos de los rasgos xeromórficos tales como: epidermis con alta densidad de células con paredes rectas más o menos engrosadas y bajo índice estomático. Presencia de una red venosa densa y abundante esclerénquima acompañando el tejido vascular y/o esclereidas idioblásticas en el mesofilo. Al comparar la anatomía de la hoja de arbustos y árboles de las Provincias Biogeográficas de las Yungas, Paranaense y Chaqueña, muchas de estas especies también habitan las Provincias del Monte y el Espinal, surge que los caracteres más variables son la distribución de los estomas en una o ambas epidermis y el tipo de mesofilo. Mientras que, el tipo de estomas y tricomas, por el contrario, son caracteres estables con valor taxonómico.

\section{Agradecimientos}

Nuestro reconocimiento al personal de los herbarios (BA, LP, LPAG, SI), quienes permiten como custodios de los ejemplares, utilizarlos para trabajos científicos. Muchas gracias a M. Alejandra Migoya por el delineado del mapa y lo hacemos extensivo a Mario Sánchez y Mariela Theiller, técnicos del Servicio de Microscopía Electrónica y, a los revisores anónimos. Este estudio se realizó en el marco del Programa de Incentivos a los docentes-investigadores, Decreto 2427/93, Secretaría de Política Universitaria, Ministerio de Educación de la Nación Argentina.

\section{Bibliografía}

ANCIBOR, E. 1992. Anatomía ecológica de la vegetación de la Puna de Mendoza. I. Anatomía foliar. Parodiana 7(1-2): 63-76.

ARAMBARRI, A. M., S. E. FREIRE, M. N. COLARES, N. D. BAYÓN, M. C. NOVOA, C. MONTI \& S. A. STENGLEIN. 2006. Leaf anatomy of medicinal 
shrubs and trees from Gallery forests of the Paranaense Province (Argentina). Part 1. Bol. Soc. Argent. Bot. 41(3-4): 233-268.

- 2008. Leaf anatomy of medicinal shrubs and trees from Misiones forest of the Paranaense Province (Argentina). Part 2. Bol. Soc. Argent. Bot. 43(1-2): 31-60.

ARAMBARRI, A. M., S. E. FREIRE, N. D. BAYÓN, M. N. COLARES, C. MONTI, M. C. NOVOA \& M. P. HERNÁNDEZ. 2009a. Morfoanatomía foliar de árboles medicinales de la Provincia Biogeográfica de las Yungas (Argentina). Bol. Latinoam. Caribe Plant. Med. Aromat. 8(5): 342-379.

2009b. Micrografía foliar de arbustos y pequeños árboles medicinales de la Provincia Biogeográfica de las Yungas (Argentina). Kurtziana 35(1): 1-31.

—, M. C. NOVOA, N. D. BAYÓN, M. P. HERNÁNDEZ, M. N. COLARES \& C. MONTI. 2011. Ecoanatomía foliar de árboles y arbustos de los distritos chaqueños occidental y serrano (Argentina). Bol. Soc. Argent. Bot. 46(3-4): 251-270.

ARENAS, P. 2003. Etnografia y alimentación entre los Toba-Nachilamole'ek y Wichi-Lhuku'tas del Chaco central (Argentina). CONICET, CEFYBO, IBODA. $19 \mathrm{pp}$.

BERNARDELLO, L. M. 1982. Estudios en Lycium (Solanaceae) I. Anatomía de hoja y tallo, y sus diferencias con Grabowskia. Bol. Soc. Argent. Bot. 21: 153-185.

BIANCO, C. A., T. A. KRAUS \& A. C. VEGETTI. 2004. La hoja. Morfología externa y anatomía. Universidad Nacional de Río Cuarto, Universidad Nacional del Litoral, REUN. 196 pp.

BILONI, J. S. 1990. Árboles autóctonos argentinos. Tipográfica Editora Argentina, Buenos Aires. 335 pp.

BURKART, A. 1952. Las leguminosas argentinas. Ed. Acme, Buenos Aires. 102 pp.

1979. Sapotaceae. En: A. BURKART (ed.), Flora ilustrada de Entre Ríos. Vol. 6: 43-47. Colección Científica INTA, Buenos Aires.

CABRERA, A. L. 1994. Regiones fitogeográficas argentinas. Enc. Argent. Agricul. y Jard. 1st. reimpresión, T 2(1): 1-85, Ed. Acme, Buenos Aires.

CARRIZO, E. DEL V., M. O. PALACIO \& L. D. ROIC (ex aequo). 2005. Uso medicinal de algunas especies nativas en Santiago del Estero (República Argentina). Dominguezia 21(1): 25-32.

CORTADI, A., O. DI SAPIO \& M. GATTUSO. 1996. Caracteres anatómicos de tres especies medicinales de la familia Myrtaceae. Acta Farm. Bonaerense 15: 109-123.

COLARES, M. N., N. D. BAYÓN, S. A. STENGLEIN \& A. M. ARAMBARRI. 1999. Anatomía y etnobotánica de las especies medicinales de la Provincia Pampeana: Solanaceae (excepto Grabowskia y Solanum). Acta Farm. Bonaerense 18: 171-182.

COSA DE GASTIAZORO, M. T. 1991. Estudio morfoanatómico de órganos vegetativos en Cestroideae (Solanaceae) I: tribu Nicotianeae. Kurtziana 21: 111152.

COSA, M. T. \& N. DOTTORI. 2010. Adaptaciones anatómicas de plantas medicinales a la diversidad de ambientes. X Simposio Argentina y XIII Simposio Latinoamericano de Farmacobotánica, Córdoba. 73 pp.

, G. BRUNO, M. HADID, L. STIEFKENS, I. LISCOVSKY, M. MATESEVACH \& N. DELBÓN. 2011. Atlas de anatomía vegetal. Tejidos y órganos vegetativos. Cátedra de Morfología Vegetal. Escuela de Biología, Facultad de Cs. Exactas, Físicas y Naturales, Universidad Nacional de Córdoba. Formato disco compacto. ISBN 978-950-33-0879-0.

CRISTÓBAL, C. L. 2007. Sterculiaceae of Paraguay. I. Ayenia, Byttneria, Guazuma, Helicteres, Melochia y Sterculia. Bonplandia 16(1-2): 5-142.

CRONQUIST, A. 1988. The evolution and classification of flowering plants. NY Botanical Garden, Bronx. $555 \mathrm{pp}$.

D’AMBROGIO DE ARGÜESO, A. 1986. Manual de técnicas en histología vegetal. Ed. Hemisferio Sur, Buenos Aires. 83 pp.

DEMAIO, P., U. O. KARLIN \& M. MEDINA 2002. Árboles nativos del centro de Argentina, Literature of Latin America, Buenos Aires. 210 pp.

DIGILIO, A. P. \& P. LEGNAME 1966. Los árboles indígenas de la provincia de Tucumán. Opera Lilloana 15: $76 \mathrm{pp}$.

DIZEO DE STRITTMATTER, C. 1973. Nueva técnica de diafanización. Bol. Soc. Argent. Bot. 15: 126-129.

FAHN,A.\&D.F.CUTLER. 1992.Xerophytes.Encyclopedia of plant anatomy. I-IX, 1-176, Gerbruder, Borntraeger, Berlín.

FREIRE, S. E., A. M. ARAMBARRI, N. D. BAYÓN, G. SANCHO, E. URTUBEY, C. MONTI, M. C. NOVOA \& M. N. COLARES. 2005. Epidermal characteristics of toxic plants for cattle from the Salado River Basin (Buenos Aires, Argentina). Bol. Soc. Argent. Bot. 40: 241-281.

GUANTAY, M. E. 2004. Morfoanatomía y arquitectura foliar de Blepharocalyx salicifolius (Kunth) O. Berg (Myrtaceae). Lilloa 41: 85-92.

HABERLANDT, G. 1914. Physiological plant anatomy, (Translated from the 4th German edition by Montagu Drummond). MacMillan, London. En Anatomy of the Dicotyledons, (Metcalfe \& Chalk, 1979). 2nd. Ed. Clarendon Press, Oxford.

HURRELL, J. A., E. A. ULIBARRI, J. P. PUENTES, F. BUET COSTANTINO, P. M. ARENAS \& M. L. POCHETTINO. 2011. Leguminosas medicinales y alimenticias utilizadas en la conurbación Buenos Aires-La Plata, Argentina. Bol. Latinoam. Caribe Plant Med Aromat 10(5): 443-455.

JUÁREZ DE VARELA, F. \& L. J. NOVARA. 2007. Anacardiaceae, en Flora del Valle de Lerma Aportes Botánicos de Salta. Serie Flora. Facultad de Ciencias 
Naturales, Universidad Nacional de Salta, 8(6): 1-28.

KELLER, H.A. 2010. Plantas colorantes utilizadas por los guaraníes de Misiones, Argentina. Bonplandia 19(1): 11-25.

\& H. F. ROMERO. 2006. Plantas medicinales de la Reserva de Biósfera Yabotí (Misiones, Argentina). Bonplandia 15(3-4): 125-141.

— - F. E. GATTI \& J. HERRERA. 2009. Novelties in Casearia (Flacourtiaceae) for Argentina. Bonplandia 18(1): 13-17.

LEGNAME, P. R. 1982. Árboles indígenas del noroeste argentino. Opera Lilloana 34: 215 pp.

LEONARDI, D., O. DI SAPIO, M. GATTUSO \& S. GATTUSO. 2002. Caracteres morfoanatómicos de la corteza y hojas de Tabebuia impetiginosa y $T$. heptaphylla (Bignoniaceae). Bol. Soc. Argent. Bot. 37: 51-61.

LICOVSKY, I. J. \& M. T. COSA. 2005. Anatomía comparada de hoja y tallo en los representantes de Cestreae G. Don (Solanaceae) de Argentina. Gayana Bot. 62: 33-43.

LORCA, G. G., A. G. AMAT \& C. GONZÁLEZ. 1995. Análisis comparativo de caracteres diagnósticos para la identificación de tres especies argentina de Myrtaceae empleadas en la medicina popular. Acta Farm. Bonaerense 14: 81-86.

MARTÍNEZ CROVETTO, R. 1964. Estudios etnobotánicos I. Nombres de plantas y su utilidad según los indios tobas del este del Chaco. Bonplandia 1(4): 279-333.

— 1967. Tintorería Toba. Bonplandia 2(5): 103-105.

. 1981. Las plantas utilizadas en medicina popular en el noroeste de Corrientes. Fundación Miguel Lillo. Tucumán. Miscelánea 69: 7-139.

METCALFE, C. R. \& L. CHALK. 1950. Anatomy of the Dicotyledons. Vol. 1, 2. Clarendon Press, Oxford. $1201 \mathrm{pp}$.

1979. Anatomy of the Dicotyledons. 2nd. Ed. Vol.

1. Clarendon Press, Oxford. 276 pp.

- 1989. Anatomy of the Dicotyledons. 2nd. Ed. Vol. 2 Clarendon Press, Oxford. 297 pp.

MICHELIN, D. C., G. C. deALMEIDA, K. J. GALINA, G. C. LOPES, C. V. NAKAMURA, B. P. DIAS FILHO, T. UEDA-NAKAMURA, H. R. N. SALGADO \& J. C. PALAZZO de MELLO. 2010. Antidiarrheal activitiy of Guazuma ulmifolia Lam. (Sterculiaceae). Lat. Am. J. Pharm. 29(6): 1024-1028.

MONTE LUCHIARI DA SILVA, A., M. F. BORTOLIN COSTA, V. GONÇALVES LEITE, A. ALVES REZENDE \& S. DE PÁDUA TEXEIRA. 2009. Anatomia foliar com implicações taxonómicas em espécies de ipês. Hoehnea 36(2): 329-338.

MUÑOZ, J.D. 2000. Anacardiaceae. Flora Fanerogámica Argentina. 65:1-28.

PEÑA-CHOCARRO, M. C., J. DE EGEA JUVINEL, M.
VERA, H. MATURO \& S. KNAPP. 2006. Guía de árboles y arbustos del Chaco húmedo, The Natural History Museum, Guyra Paraguay, Fundación Moisés Bertoni y Fundación Hábitat y Desarrollo: Asunción. $223 \mathrm{pp}$.

PEREA, M., G. PEDRAZA \& J. DEL VALLE LUCEROS. 2007. Relevamiento de la flora arbórea autóctona en la provincia de Catamarca. Consejo Federal de Inversiones, Ciudad Autónoma de Buenos Aires y Gobierno de la provincia de Catamarca. 312 pp.

RIGONATTO, O., N. DOTTORI \& M. T. COSA. 2005. Anatomía en Solanum argentinum (Solanaceae). Kurtziana 31(1-2): 21-28.

RUÍZ, A. I., M. I. MERCADO, M. E. GUANTAY \& G. I. PONESSA. 2009. Morfoanatomía y arquitectura foliar de Schinus areira (Anacardiaceae). Lilloa 46(1-2): 137-146.

ROTH, I. 1984. Stratification of tropical forests as seen in leaf structure. Junk Publ., The Hague, Boston Lancaster. $522 \mathrm{pp}$.

1990. Leaf structure of a Venezuela cloud forest (in relation to the microclimate). In: I. ROTH (ed.), Encyclop. Plt. Anat. Vol 14, Part 3, Gebruder Borntraeger, Berlín. 242 pp.

1995. Leaf structure: montane regions of Venezuela with an excursion into Argentina. In. I. ROTH (ed.), Encyclop. Plt. Anat. Vol 14, Part 3, Gebruder Borntraeger, Berlín. 249 pp.

SALISBURY, E. 1929. On the causes and ecological significance of stomatal frequency with special reference to the Woodland flora. Phil. Trans. R. Soc. Lond., Ser B 216: 1-65.

SCHNEIDER, N. F. Z., N. F. DE MOURA, L. C. MENDONÇA \& R. B. N. DENARDIM. 2010. Atividade antimicrobiana das folhas de Casearia sylvestris Swart. Lat. Am. J. Pharm. 29(4): 631-634.

SILVA, A. M. L., M. F. B. COSTA, V. G. LEITE, A. A. REZENDE \& S. P. TEIXEIRA. 2009. Leaf anatomy with taxonomic implications in "ipe" species. Hoehnea 36(2): 329-338.

STACE, A. 1965. Cuticular studies as an aid to plant anatomy. Bull. Br. Mus. (Nat. Hist.) Bot. 4: 1-78.

STENGLEIN, S.A., A. M. ARAMBARRI, M. N. COLARES, M. C. NOVOA \& C.E. VIZCAÍNO. 2003. Leaf epidermal characteristics of Lotus subgenus Acmispon (Fabaceae: Loteae) and a numerical taxonomic evaluation. Canad. J. Bot. 81: 933-944.

TOURSARKISSIAN, A. 1980. Plantas medicinales de la Argentina. Ed. Hemisferio Sur, Buenos Aires. 178 pp.

ZULOAGA, F. O., O. MORRONE \& M. J. BELGRANO (eds.). 2008. Catálogo de las plantas vasculares del Cono Sur (Argentina, Sur de Brasil, Chile, Paraguay y Uruguay). II. Monogr. Syst. Bot. Missouri Bot. Gard. 107: 1905-1908. http://www.darwin.edu.ar/ (mayo 2010). 
Tabla 1. Densidades por $\mathrm{mm}^{-2}$ de células epidérmicas, estomas y tricomas e índice estomático.

\begin{tabular}{|c|c|c|c|c|c|c|c|}
\hline TAXONES & $\begin{array}{c}\text { Células } \\
\text { epid. } \\
\text { adx }\end{array}$ & $\begin{array}{c}/ \mathbf{m m}-\mathbf{2} \\
\mathbf{a b x}\end{array}$ & $\begin{array}{c}\text { Estomas } \\
\text { adx }\end{array}$ & $\begin{array}{c}/ \mathbf{m m}-\mathbf{2} \\
\mathbf{a b x}\end{array}$ & Tricomas/mm-2 & $\begin{array}{c}\text { Índice } \\
\text { adx }\end{array}$ & $\begin{array}{c}\text { estomático } \\
\text { abx }\end{array}$ \\
\hline Allophylus edulis & & 7437,5 & & 950,1 & baja densidad & & $11,50 \%$ \\
\hline $\begin{array}{l}\text { Anadenanthera colubrina var. } \\
\text { cebil }\end{array}$ & 5940,7 & 3939,4 & 289,1 & 1236,1 & baja densidad & $7,12 \%$ & $23,31 \%$ \\
\hline Aspidosperma quebracho-blanco & 14787,9 & 17181,8 & 111,7 & 123,1 & 84,28 & $0,68 \%$ & $0,75 \%$ \\
\hline Astronium balansae & & 4986,7 & & 422,3 & baja densidad & & $9 \%$ \\
\hline Caesalpinia paraguariensis & 2096,2 & 2704,6 & 72,2 & 477,3 & 15,1 & $3,99 \%$ & $14,84 \%$ \\
\hline Carica quercifolia & & 2400,8 & & 159,5 & glabra & & $6,23 \%$ \\
\hline Casearia sylvestris & & 6783,1 & & 1409,1 & baja densidad & & $17,27 \%$ \\
\hline Chrysophyllum marginatum & & 4467,8 & & 268,9 & glabra & & $5,70 \%$ \\
\hline Enterolobium contortisiliquum & & 7010,6 & & 715,1 & 29 & & $10,65 \%$ \\
\hline Erythrina dominguezii & 2151,5 & 3400 & 166,7 & 439,4 & 60,6 & $7,18 \%$ & $11,54 \%$ \\
\hline Eugenia uniflora & & 4491,5 & & 892,9 & glabra & & $16,87 \%$ \\
\hline Genipa americana & & 1740,4 & & 354,1 & glabra & & $16,90 \%$ \\
\hline Geoffroea decorticans & 4792,4 & 4661,3 & 484,8 & 358,3 & 78,1 & $9,15 \%$ & $7,12 \%$ \\
\hline Grabowskia duplicata & & 3032,3 & & 121,2 & baja densidad & & $3,84 \%$ \\
\hline $\begin{array}{l}\text { Guarea macrophylla subsp. } \\
\text { spicaeflora }\end{array}$ & & 4232,2 & & 310,6 & 22,7 & & $6,47 \%$ \\
\hline Guazuma ulmifolia var. ulmifolia & & 6160,9 & & 1127,8 & 53,1 & & $15,47 \%$ \\
\hline Handroanthus heptaphyllus & & 4812,5 & & 393,9 & 22,1 & & $7,57 \%$ \\
\hline Holocalyx balansae & & 4177,3 & & 465,1 & baja densidad & & $10,11 \%$ \\
\hline Maytenus ilicifolia & & 2630,5 & & 392,1 & glabra & & $12,68 \%$ \\
\hline Myrcianthes pungens & & 2384,4 & & 464,9 & baja densidad & & $16,76 \%$ \\
\hline Ocotea diospyrifolia & & 5615,6 & & 420,4 & baja densidad & & $6,43 \%$ \\
\hline Patagonula americana & & 6909,1 & & 632,5 & baja densidad & & $8,48 \%$ \\
\hline Peltophorum dubium & & 7543,9 & & 611,1 & 50,5 & & $7,70 \%$ \\
\hline Pouteria gardneriana & & 5636,4 & & 388,3 & 30,3 & & $6,44 \%$ \\
\hline Prosopis affinis & 4402,3 & 4357,1 & 677,3 & 325,8 & 257,6 & $13,59 \%$ & $7,38 \%$ \\
\hline Prosopis alba & 4161,6 & 4137,9 & 690,4 & 346,5 & 309,6 & $14,08 \%$ & $7,75 \%$ \\
\hline Prosopis kuntzei & 3114,9 & 3762,3 & 467,2 & 517,1 & 287,8 & $12,49 \%$ & $11,25 \%$ \\
\hline Prosopis nigra & 3447,1 & 3221,1 & 500,1 & 299,1 & 174,2 & $13,45 \%$ & $8,48 \%$ \\
\hline Pterogyne nitens & & 6582,3 & & 662,8 & baja densidad & & $9,04 \%$ \\
\hline Rollinia emarginata & & 2415,1 & & 329,6 & baja densidad & & $10,71 \%$ \\
\hline Salix humboldtiana & 4284,1 & 4126,9 & 220,3 & 268,9 & 11,33 & $5,11 \%$ & $6,05 \%$ \\
\hline Sapindus saponaria & & 8764,5 & & 817,5 & 11,4 & & $8,07 \%$ \\
\hline Tabebuia aurea & 5518,9 & 7295,4 & 58,3 & 393,9 & 356,1 & $1,02 \%$ & $5,12 \%$ \\
\hline Tabernaemontana catharinensis & & 3142,1 & & 351,3 & glabra & & $10,39 \%$ \\
\hline Terminalia triflora & & 4093,7 & & 469,6 & 24,5 & & $10,43 \%$ \\
\hline Trichilia catigua & & 6299,2 & & 384,4 & 15,1 & & $5,75 \%$ \\
\hline
\end{tabular}




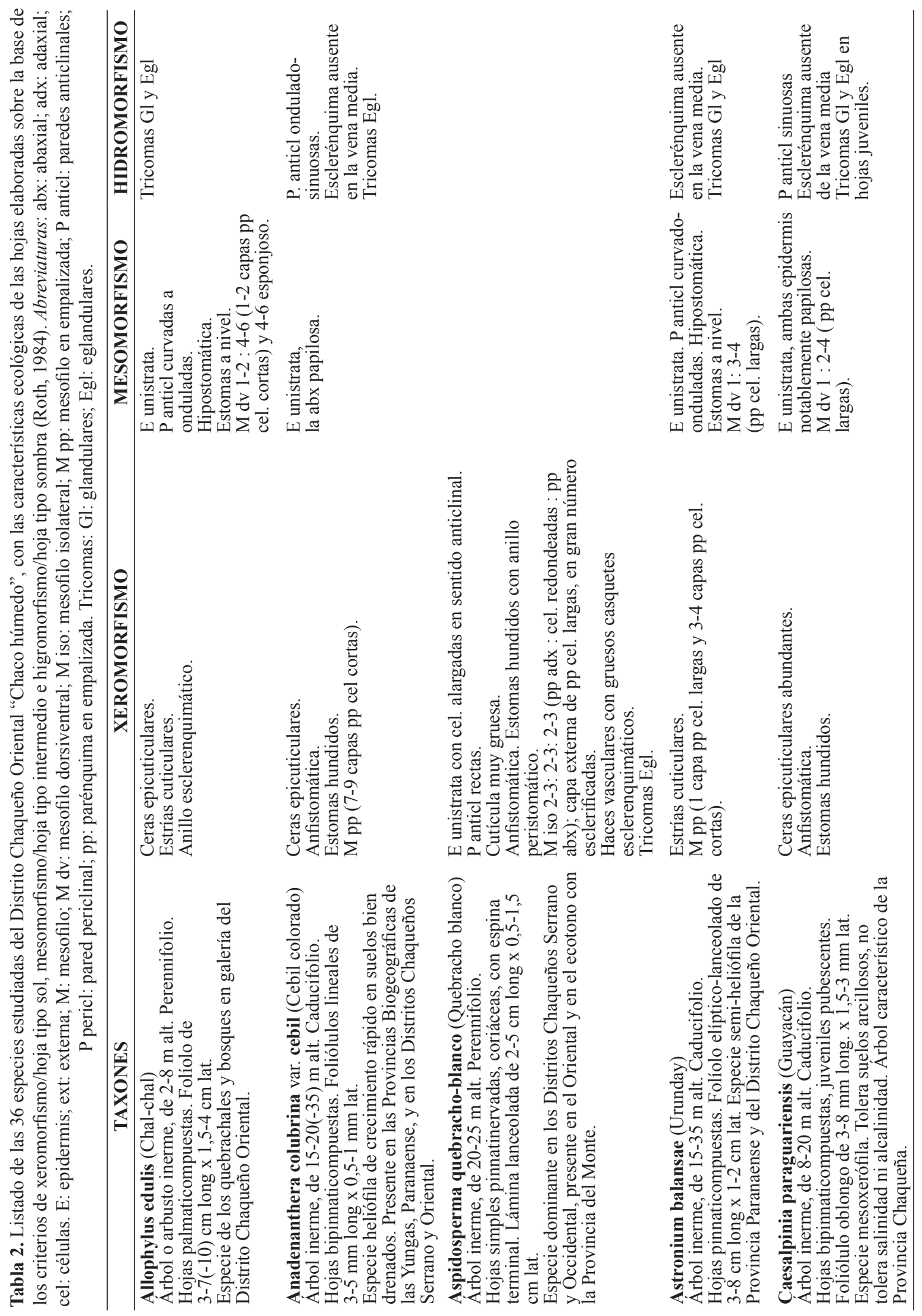




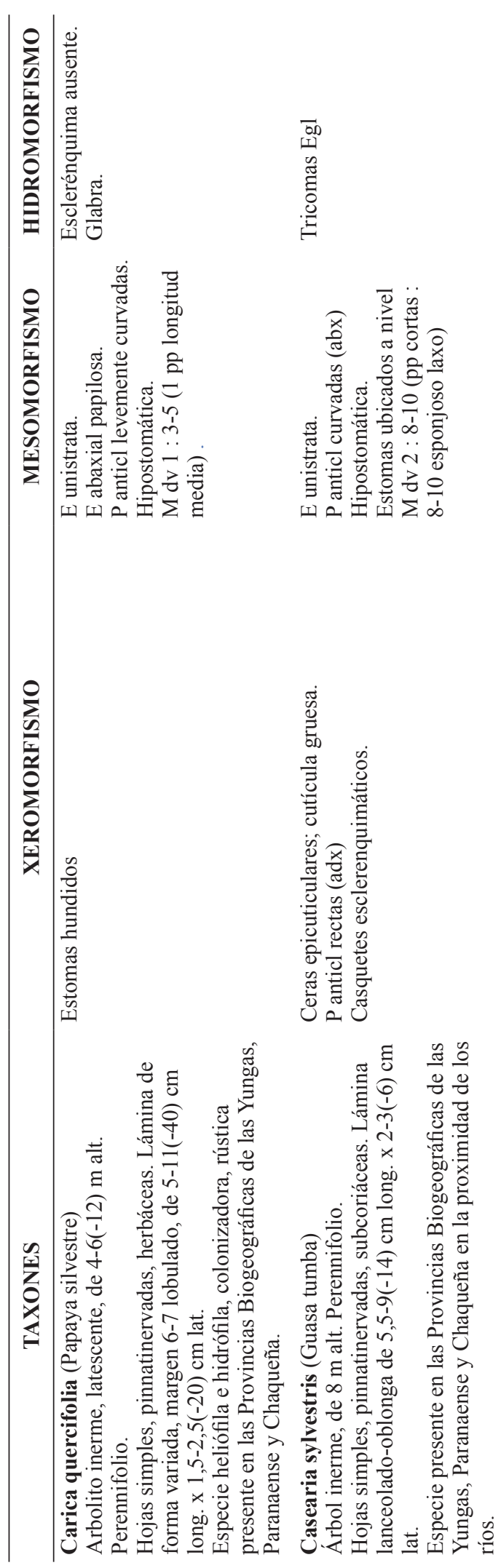

$\frac{\pi}{\frac{\pi}{0}}$
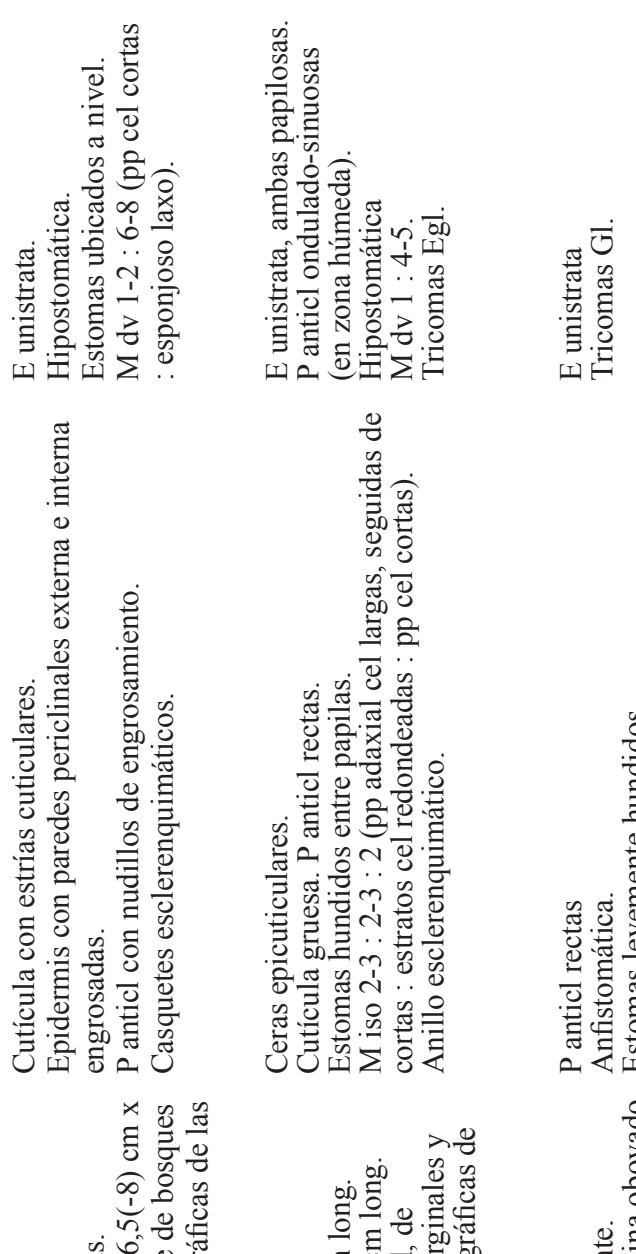

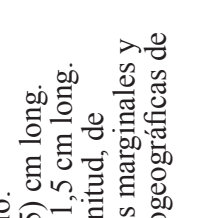

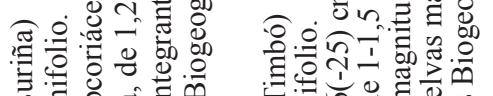

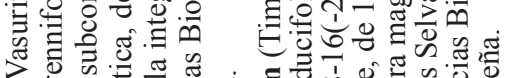

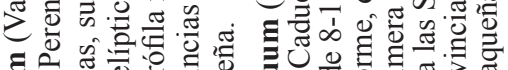

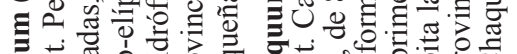

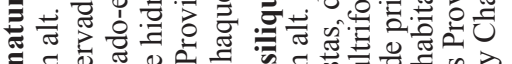

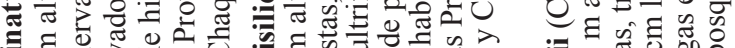

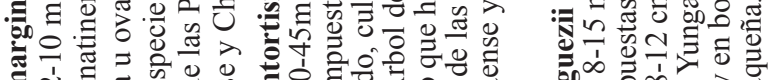

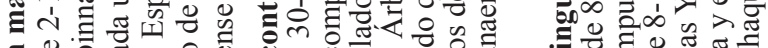

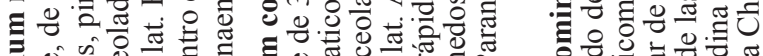

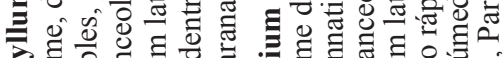

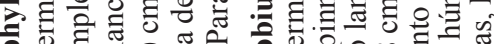

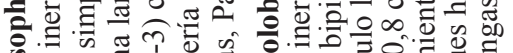

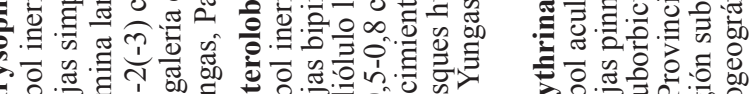

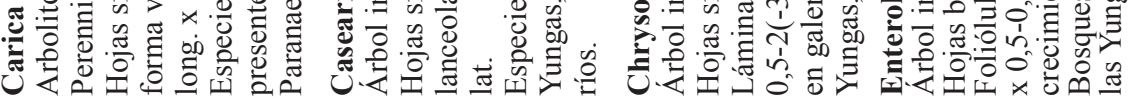

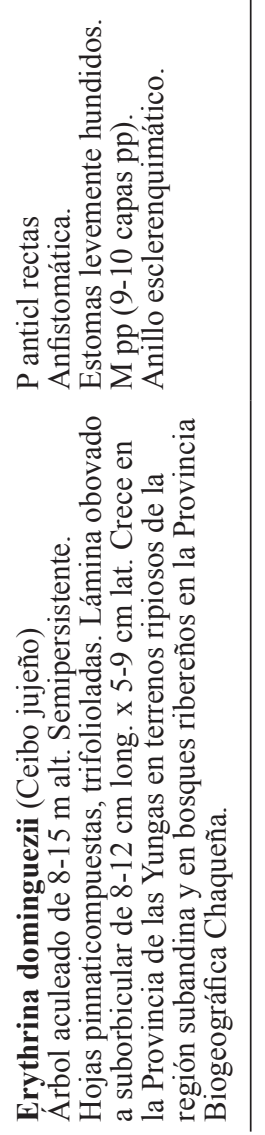




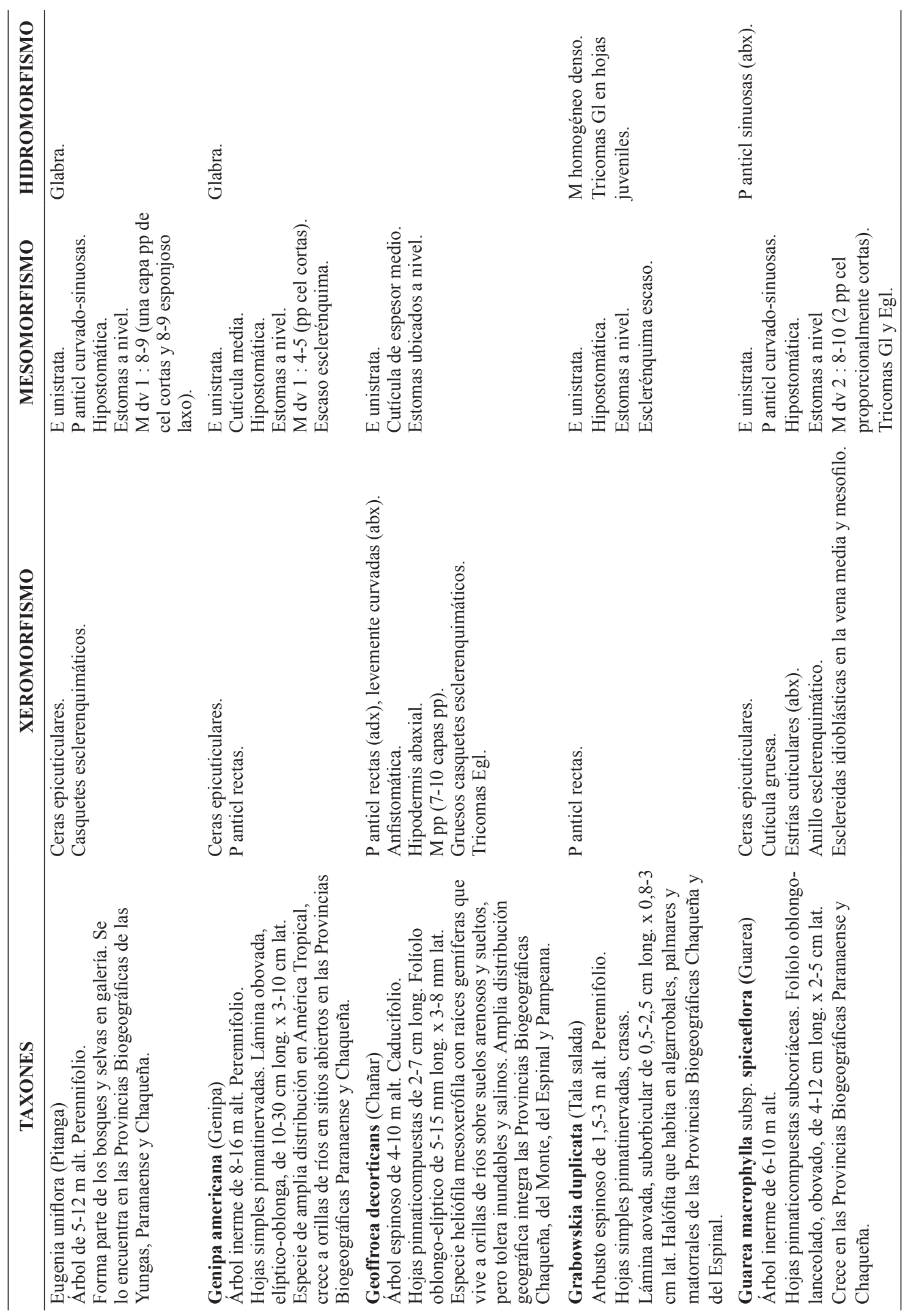




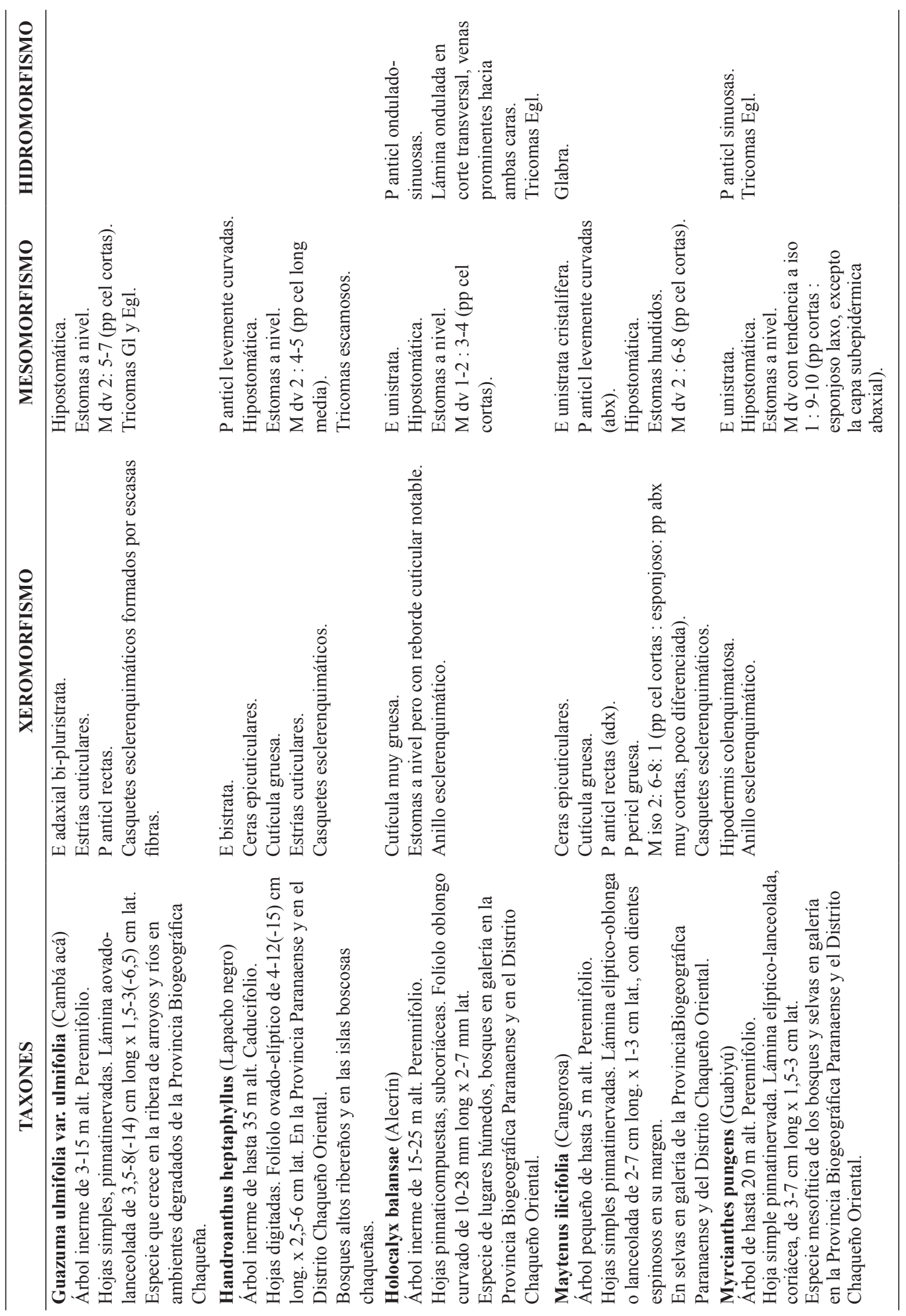




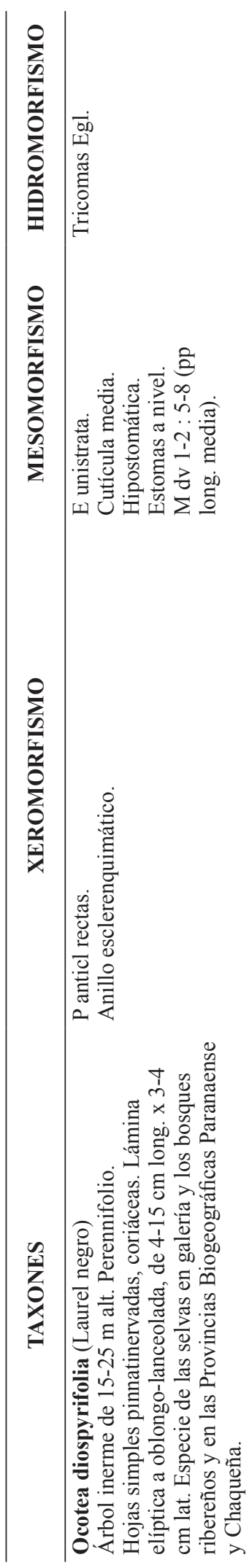

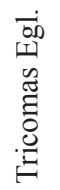

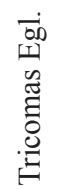
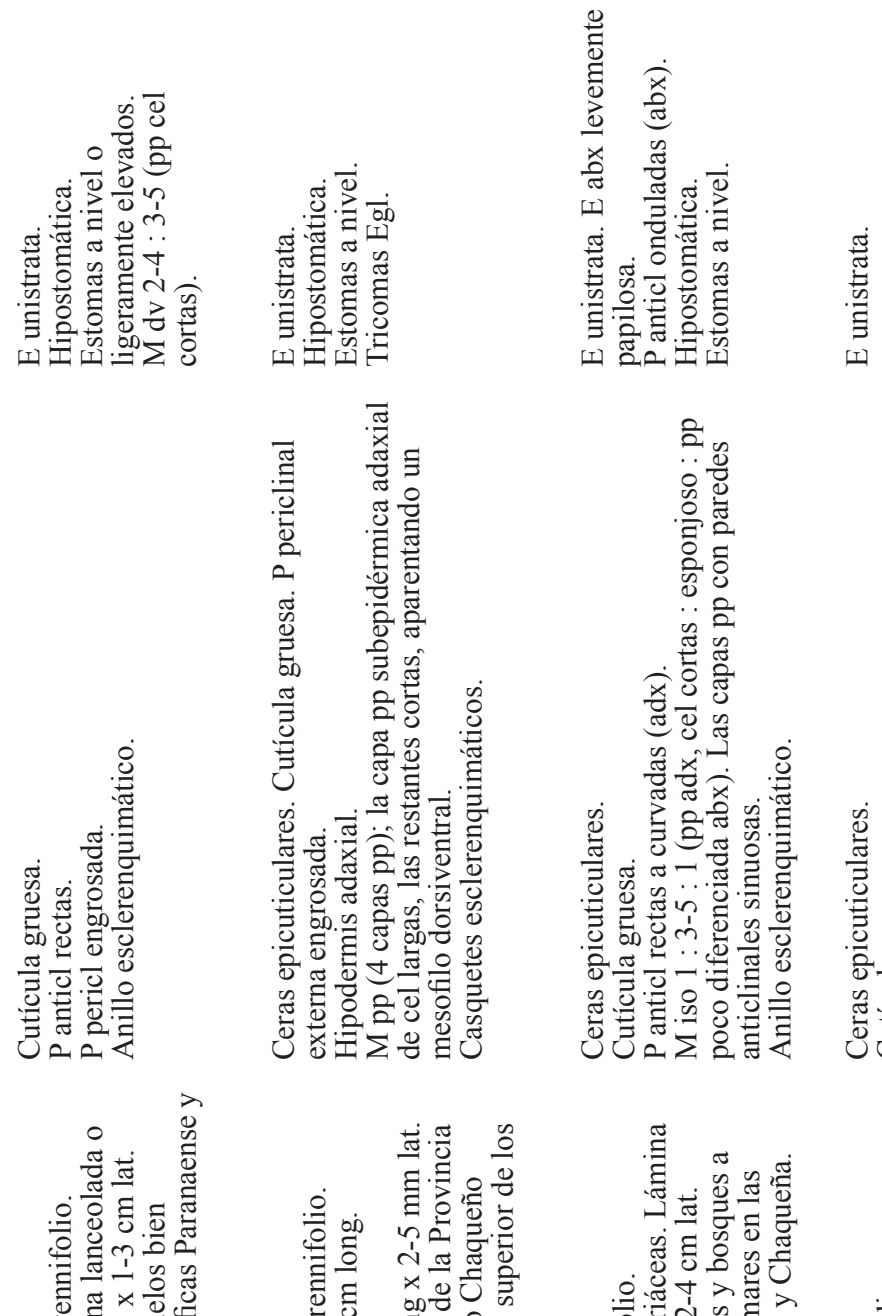

竝

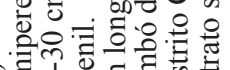

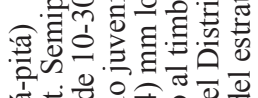

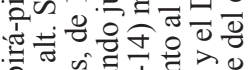

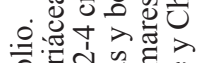

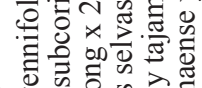

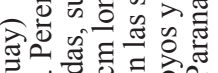

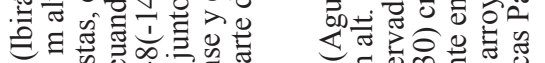

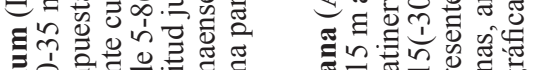

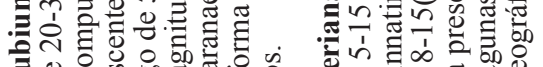
三. घ 0 .

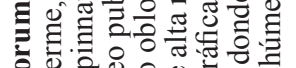
을.

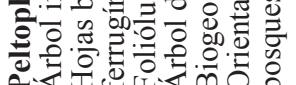

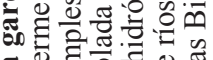

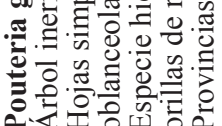

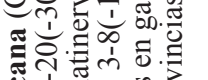

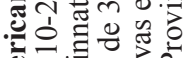

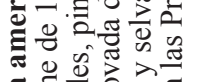

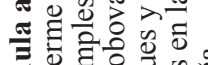

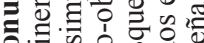

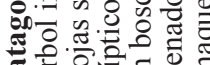

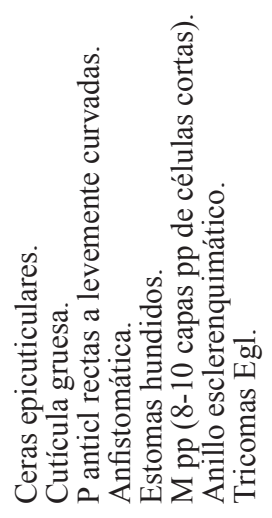

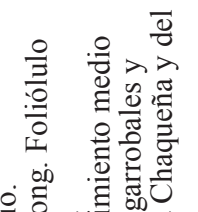

莺

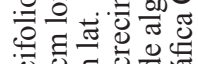

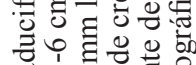

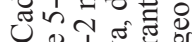

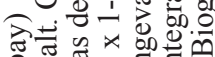

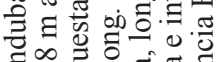

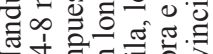
乙o no ส.

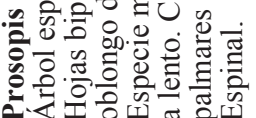



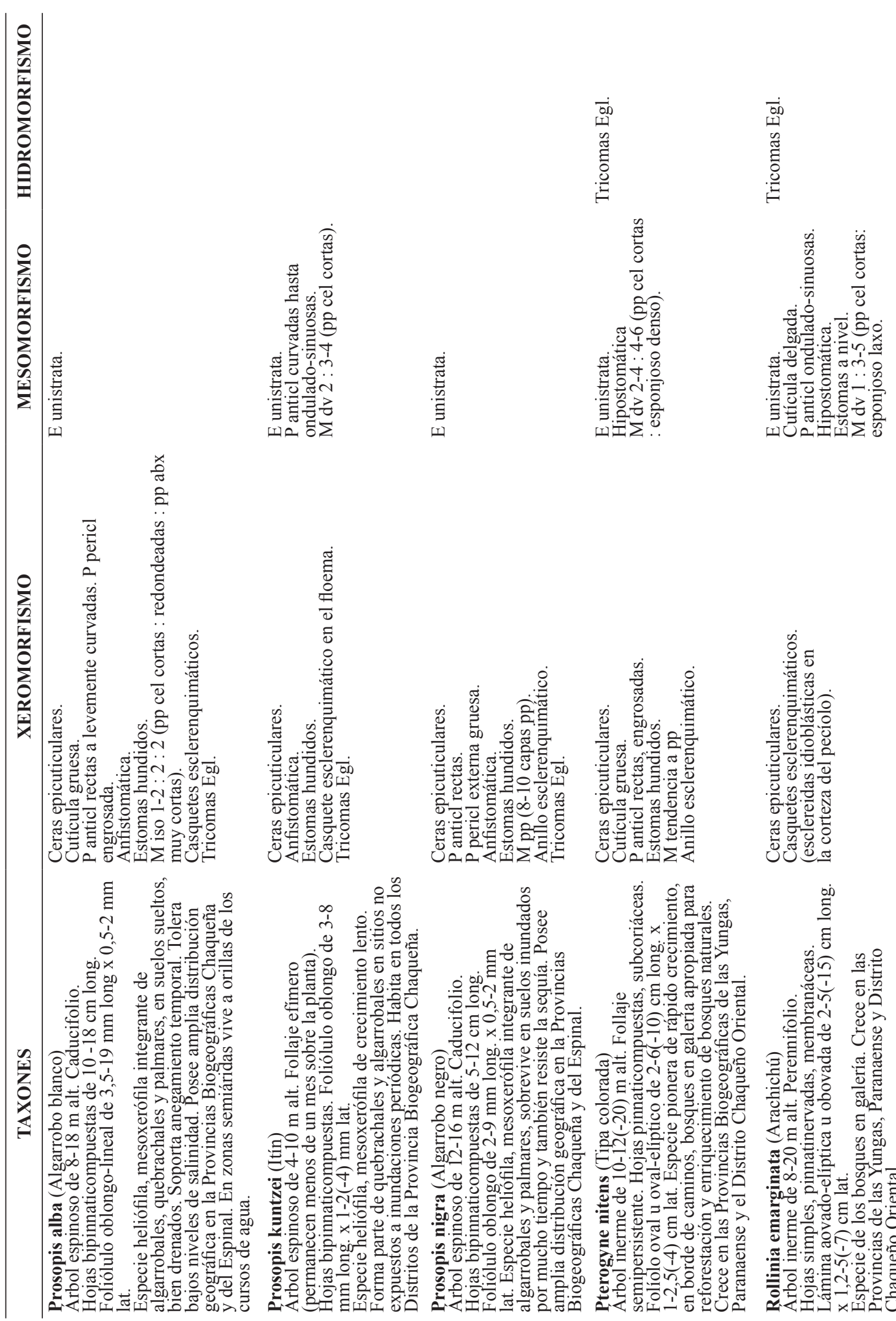

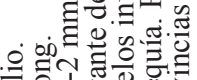

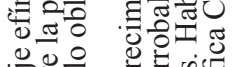

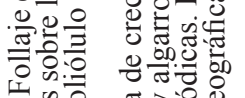

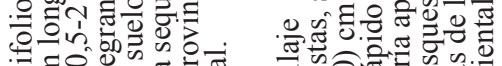

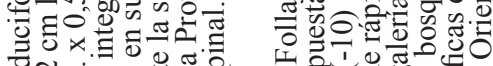
4.

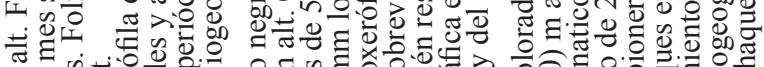

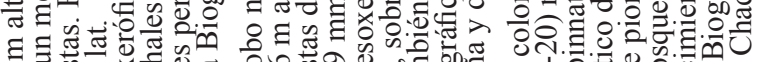

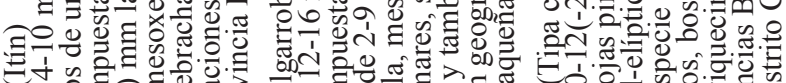

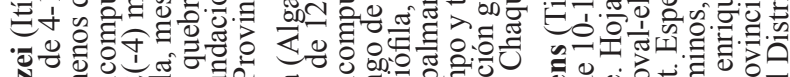

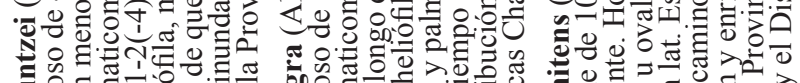
互.

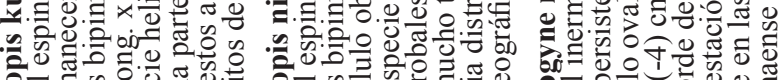

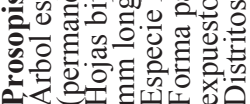
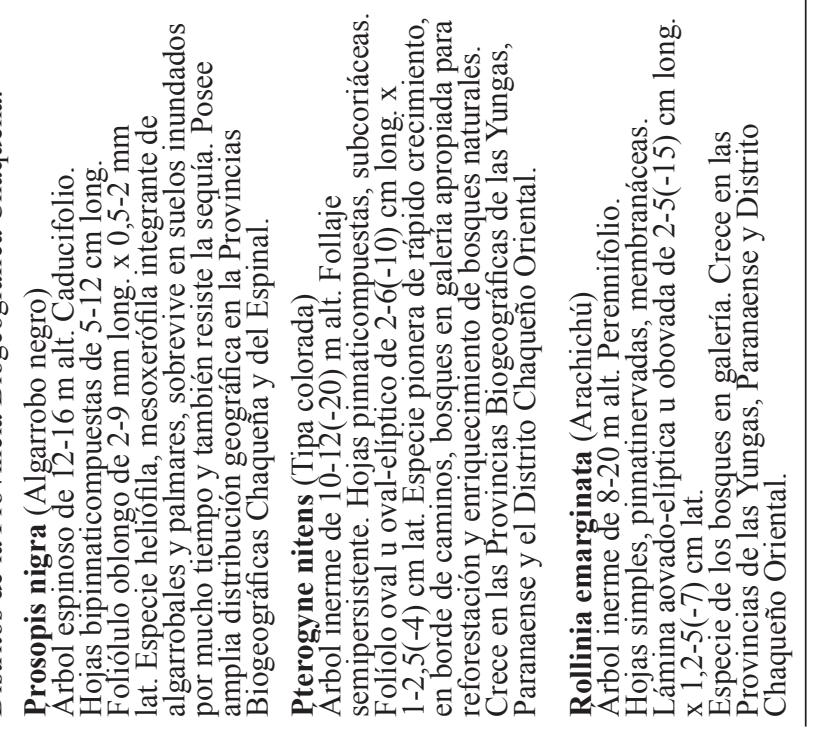


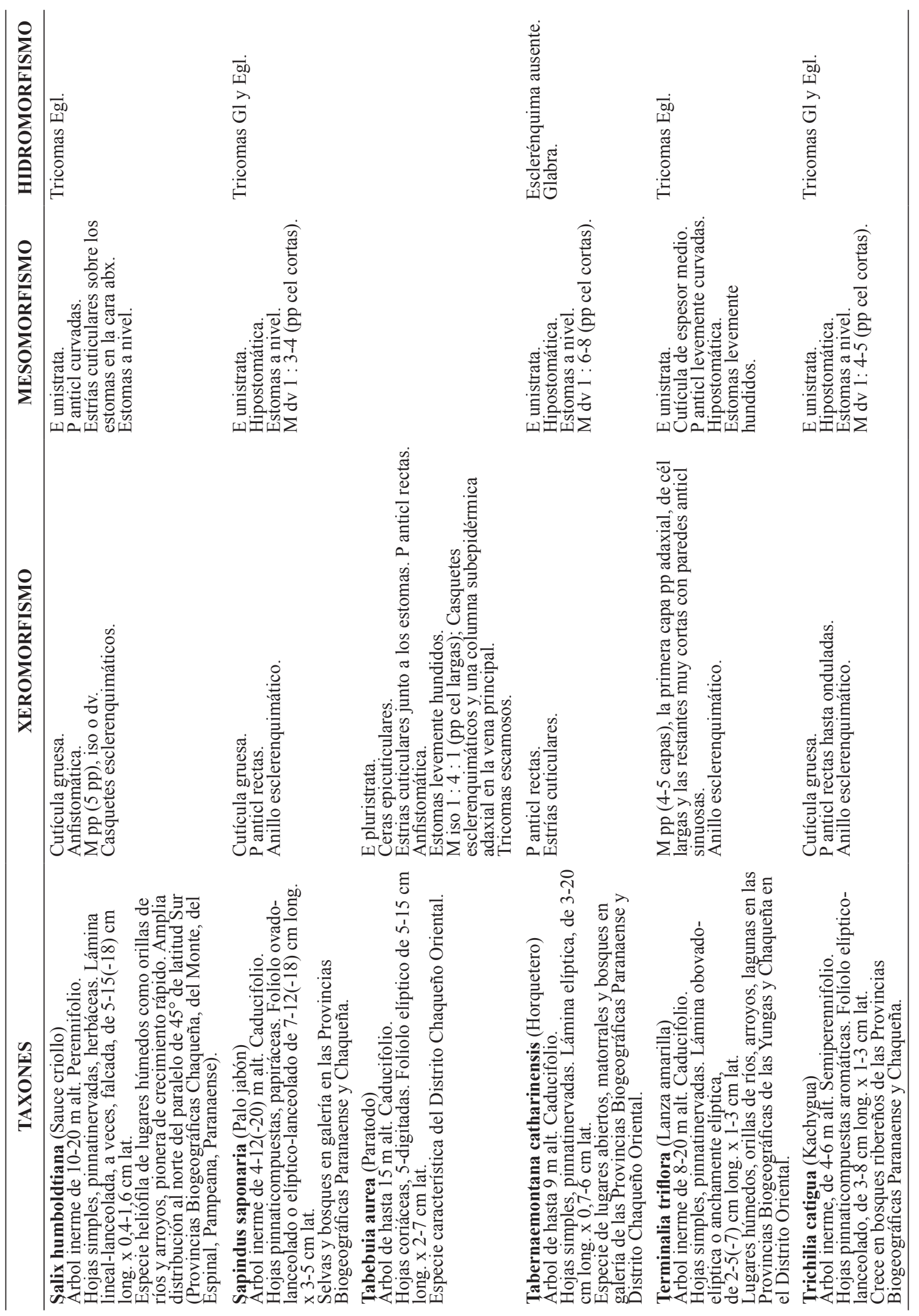


Apéndice 1. Lista alfabética de las 36 especies estudiadas con la familia y los materiales testigos indicando localidades y coleccionistas

1. Allophylus edulis (A. St.-Hil.,A. Juss. \& Cambess) Hieron. ex Niederl. (Sapindaceae). Corrientes: Dep. Itatí, Ramada Paso, 1-XI-1970, Krapovickas \& Cristobal 16469 (LP). Misiones: Dep. Iguazú, Parque Nacional Iguazú, 21-IX-1970, Dimitri \& Amorín 91 (LPAG); 21-X-1970, Dimitri \& Amorín s.n. (LPAG 1227). 2. Anadenanthera colubrina (Vell.) Brenan var. cebil (Griseb.) Reis (Leguminosae). Buenos Aires: Pdo. La Plata, La Plata, Jardín Botánico y Arboretum "C. Spegazzini", Fac. Cs. Agrarias y Forestales, UNLP, I-2011, Arambarri s.n. (LPAG). Chaco: Dep. Sargento Cabral, Loc. no citada, 15-XI-1998, Delucchi \& al., 1977 (LP). Jujuy: Dep. Tafí, Yerba Buena, 2-XI1920, Venturi s.n. (BA). Salta: Dep. Capital, Cerro San Bernardo, 6-XI-1994, Delucchi 813 (LPAG). Santiago del Estero: Dep. Guasayán, sierras de Guasayán, 17-XI-1998, Delucchi \& al. 2079 (LP). 3. Aspidosperma quebracho-blanco Schltdl. (Apocynaceae). Formosa: Dpto. Bermejo, Laguna Yema, 22-IX-2004, Bayón \& Moreno 845 (LPAG). Santiago del Estero: Dep. Moreno, Quimilí, 19-VI2010, Mengascini s.n. (LPAG). 4. Astronium balansae Engl. (Anacardiaceae). Santa Fe: Dep. General Obligado, San Antonio de Obligado, 10-I1937, Ragonese 2413 (LP). Corrientes: Dep. San Luis del Palmar, San Luis del Palmar, 28-XII-1967, Pedersen 8736 (LP). 5. Caesalpinia paraguariensis (D. Parodi) Burkart (Leguminosae). Buenos Aires: Pdo. La Plata, La Plata, Jardín Botánico y Arboretum "C. Spegazzini", Fac. Cs. Agrarias y Forestales, UNLP, I-2011, Arambarri s.n. (LPAG). Chaco: Dpto Tapenagá, Enrique Urién, XI-1940, Rodrigo 2709 (LP 040935). Formosa: Dep. Bermejo, Laguna Yema, 17-V-2004, Bayón \& Moreno 725 (LPAG). Santiago del Estero: Dep. Guasayán, Guasayán, 17-XI-1998, Delucchi \& al. 2064 (LP). 6. Carica quercifolia (A. St.-Hil.) Hieron. (Caricaceae). Corrientes: Dep. Mburucuyá, Mburucuyá, 15-IV-1952, Pedersen 1678 (LP 892409). Chaco: Dep. Primero de Mayo, Colonia Benítez, 08-I-1965, Schulz 14309 (LP). 7. Casearia sylvestris Sw. (Salicaceae). Corrientes: Dep. Capital, Riachuelo, 8-VIII-1973, Schinini, Tressens \& Benítez 6905 (LP); Dep. Mburucuyá, Mburucuyá, 6-IX-1949, Pedersen 419 (LP). Misiones: Dep. Iguazú, Parque Nacional Iguazú, 14-IX-1970, Dimitri \& Amorin 18 (LPAG). 8. Chrysophyllum marginatum (Hook. \& Arn.) Radlk. (Sapotaceae). Buenos Aires: Pdo. La Plata, La Plata, Facultad de Ciencias Agrarias y Forestales, UNLP, 8-XI-2005, Arambarri 253 (LPAG). Corrientes: Dep. Concepción, Loc. no mencionada, 4-V-1974, Petersen 1072 (LP). Chaco: Dep. Primero de Mayo, Colonia Benítez, 29-VI-1952, Schulz 8338 (LP); Dep. Primero de Mayo, Colonia Benítez, IX-1945, Meyer s.n. (LP 021679). 9. Enterolobium contortisiliquum (Vell.) Morong (Leguminosae). Buenos Aires: Pdo. La Plata, La Plata, Fac. Cs. Agrarias y Forestales, UNLP, I-2011, Arambarri s. n. (LPAG). Chaco: Dep. Tapenagá, Enrique Urién, XI-1940, Rodrigo 2709 (LP 040935). Formosa: Dep. Bermejo, Laguna Yema, 17-V-2004, Bayón \& Moreno 725 (LPAG). Santiago del Estero, Dep. Guasayán, Guasayán, 17-XI-1998, Delucchi \& al. 2064 (LP). 10. Erythrina dominguezii Hassl. (Leguminosae). Corrientes: Dep. Capital, Capital, 23-X-1970, Mariña 142 (LP). 11. Eugenia uniflora L. (Myrtaceae). Buenos Aires: Pdo. La Plata, La Plata, Arboretum, Facultad de Agronomía, 25-VI1966, Amorín s.n. (LPAG); Jardín Botánico y Arboretum "C. Spegazzini", Facultad de Ciencias Agrarias y Forestales, UNLP, 8-VII-2004, Martínez 16 (LPAG). Corrientes: Dep. Empedrado, Loc no citada, 25-X-1968, Petersen 6616 (LP). Misiones: Dep. Eldorado, Eldorado, 25-III-2005, Martínez 82 (LPAG). 12. Genipa americana L. (Rubiaceae). Chaco: Dep. Bermejo, Isla brasilera junto a la Isla del Cerrito en la confluencia del río Paraguay con el río Paraná, 20-I-1963, Schulz 12139 (SI, BA 73804). Formosa: Dep. Formosa, Formosa, XII-1917, Jörgensen 2089 (BA 5563). 13. Geoffroea decorticans (Gillies ex Hook \& Arn.) Burkart (Leguminosae). Catamarca: Dep. Belen, La Ciénaga, 13-III-1974, Diantoni \& al. 29 (LP). Jujuy: Dep. Purmamarca, Loc. no citada, 12-II1993, Cabrera 15055 (LP). Santiago del Estero: Dep. y Loc. no citados, I-1919(LP). 14. Grabowskia duplicata Arn. (Solanaceae). Santa Fe: Dep. Vera, Santa Lucía, 24-III-1965, Tedone 5014 (LP). 15. Guarea macrophylla Vahl subsp. spicaeflora (A. Juss.) T. D. Penn. (Meliaceae). Misiones: Dep. Iguazú, Puerto Nacional Iguazú, 6-XII-1970, Volkart s.n.(LPAG 2942); X-1975, Volkarts.n.(LPAG2944). Santa Fe: Dep. General Obligado, isla La Invernada, próxima a Reconquista, 11-XII-1948, Cabrera 10463 (LP). 16. Guazuma ulmifolia Lam. var. ulmifolia (Sterculiaceae). Chaco: Dep. Bermejo, Gral. Vedia, X-1939, Schulz s.n. (LP 052995). 17. Handroanthus heptaphyllus (Vell.) Mattos (Bignoniaceae). Buenos Aires: Pdo. La Plata, La Plata, Jardín Botánico y Arboretum "C. Spegazzini”, Facultad de Ciencias Agrarias y Forestales, 15-X1992, Volkart s.n. (LPAG 2941). Misiones: Dep. Eldorado, Eldorado, 23-III-2003, Martínez 41 (LPAG 3568). Corrientes: Dep. Capital, Isla Meza, XI-1936, Rodrigo 866 (LP). 18. Holocalyx balansae Micheli (Leguminosae). Corrientes: Dep. Goya, Goya, 19-II-1970, Carnevali 1935 (SI); Dep. Santo Tomé, Ruta 38 a $17 \mathrm{~km}$ al NE de la Ruta 14, 7-XII1980, Tressens 1221 (SI). 19. Maytenus ilicifolia (Schrad.) Planch. (Celastraceae). Buenos Aires: Pdo. La Plata, La Plata, 20-VII-2006, Arambarri 262 (LPAG). Chaco: Dep. Resistencia, Loc. no 
citada, 15-IX-1956, Petersen 3983 (LP). Misiones: Dep. Montecarlo, Loc. Montecarlo, 26-III-05, Martínez 53 (LPAG). 20. Myrcianthes pungens (O. Berg) D. Legrand (Myrtaceae). Buenos Aires: Pdo. La Plata, La Plata, 24-III-1966, Amorín 148 (LPAG); Ciudad Autónoma de Buenos Aires, Capital Federal, 7-I-1991, Orfila s. n. (LPAG 4806). Corrientes: Dep. San Luis del Palmar, Loc. no citada, 29-I-1972, Mroginski, Quarin \& González 519 (LP). 21. Ocotea diospyrifolia (Meisn.) Mez (Lauraceae). Misiones: Dep. Iguazú, Parque Nacional Iguazú, 18-XI-1969, Hualde s. n. (LPAG 1378). Corrientes: Dep. Capital, Perichón, 6-VIII-1973, Schinini, Arbo \& Ferraro 6874 (LP). 22. Patagonula americana L. (Boraginaceae). Buenos Aires: Pdo. La Plata, La Plata, Jardín Botánico y Arboretum "C. Spegazzini", Facultad de Ciencias Agrarias y Forestales, 1969, Hualde s.n. (LPAG 4461); XI-2005, Monti 28 (LPAG). Misiones: Dep. Iguazú, Parque Nacional Iguazú, XI-1972, Dimitri s. n. (LPAG 4451). Corrientes: Dep. Mburucuyá, Estancia Sta. Teresa, 14-XI-1951, Pedersen 1318 (LP). 23. Peltophorum dubium (Spreng.) Taub. (Leguminosae). Buenos Aires: Pdo. La Plata, La Plata, Jardín Botánico y Arboretum "C. Spegazzini", Facultad de Ciencias Agrarias y Forestales, UNLP, I-2011, Arambarri s.n. (LPAG). Chaco: Dep. Primero de Mayo, Colonia Benítez, VII-1932, Schulz 14441 (LP). Chaco: Dep. no citado, Loc. no citada, Malabroza, XI-1893 (LP 24338). 24. Pouteria gardneriana (A. DC.) Radlk.; Dep. Fontana, Colonia Benítez, 2-X-1971, Schulz 17856 (LP). Misiones: Dep. Candelaria, Loreto, 2-XI-1931, Gruner 1050 (LP). 25. Prosopis affinis Spreng. (Leguminosae). Corrientes: Dep. Capital, Perichón, 18-XII-1975, Schinini \& Martínez Crovetto 12255 (LP). Chaco: Dep. Tapenagá, Enrique Urién, XI- 1940, Rodrigo 2403 (LP). Formosa: Dep. Bermejo, Laguna Yema, 15-XII2004, Bayón \& Moreno 963 (LPAG). 26. Prosopis alba Griseb. (Leguminosae). Córdoba: Dep. Capital, Córdoba, I-1950, Hunziker 2648 (LP). Formosa: Dep. Bermejo, Laguna Yema, 14-XII2004, Bayón \& Moreno 924 (LPAG). Santiago del Estero: Dep. y Loc., no citados, 24-IX-1944, Maldonado Bruzzone 1322 (LP 078659). 27.

Prosopis kuntzei Harms ex Kuntze (Leguminosae).

Buenos Aires: Pdo. La Plata, La Plata, Jardín Botánico y Arboretum "C. Spegazzini", Fac. Cs. Agrarias y Forestales, UNLP, I-2011, Arambarri s.n. (LPAG). Formosa: Dep. Bermejo, Laguna Yema, IX-2004, Bayón \& Moreno 874 (LPAG). 28. Prosopis nigra (Griseb.) Hieron. (Leguminosae). Buenos Aires: Pdo. La Plata, La Plata, Jardín Botánico y Arboretum "C. Spegazzini”, Fac. Cs. Agrarias y Forestales, UNLP, I-2011, Arambarri s. n. (LPAG). Córdoba: Dep. Santa María, Alta Gracia, II-1939, Rodrigo 2097 (LP). Formosa: Dep. Bermejo, Laguna Yema, 22-IX-2004, Bayón \& Moreno 831 (LPAG). Santiago del Estero: Dep. Robles, Beltrán, 22-IX-1940, Maldonado Bruzzone s. n. (LP 041368); Dep. Moreno, Quimilí, 19-VI2010, Mengascini s. n. (LPAG). 29. Pterogyne nitens Tul. (Leguminosae). Buenos Aires: Pdo. La Plata, La Plata, Jardín Botánico y Arboretum "C. Spegazzini", Facultad de Ciencias Agrarias y Forestales, Universidad Nacional de La Plata, 16VI-2006, Arambarri 260 (LPAG). Jujuy: Dep. Rosario de la Frontera, El Naranjo, 13-VIII-1927, Venturi 5224 (LP). Formosa: sin datos (LP 013842). 30. Rollinia emarginata Schltdl. (Annonaceae). Buenos Aires: Pdo. La Plata, La Plata, 16-X-2006, Arambarri 263 (LPAG). Corrientes: Dep. Empedrado, Loc. no citada, 12-X-1979, Pedersen 12487 (LP); Dep. Santo Tomé, Colonia Garabí, 3-XII-1970, Krapovickas \& al. 17025 (LP). 31. Salix humboldtiana Willd. (Salicaceae). Buenos Aires: Pdo. La Plata, Isla Martín García, 16-XII1991, Hurrell \& al. 948 (LP); La Plata, XI-2004, Monti 27 (LPAG). Corrientes: Dep. Capital, Corrientes, 15-VIII-1971, Krapovickas \& Cristobal 19597 (LP). 32. Sapindus saponaria L. (Sapindaceae). Buenos Aires: Pdo. La Plata, La Plata, Jardín Botánico y Arboretum "C. Spegazzini", Facultad de Ciencias Agrarias y Forestales, UNLP, X-2007, Colares \& Arambarri s. n. (LPAG); s. f., Montaldi 382 (LPAG 2600). Entre Ríos: Dep. Paraná, Paraná, 15-III-1990, Orfila s.n. (LPAG 2601). Corrientes: Dep. Capital, Corrientes, 21VII-1976, Tressens, Schinini, Benitez \& Ahumada 757 (LP). 33. Tabebuia aurea (Silva Manso) Benth. \& Hook. f. ex S. Moore (Bignoniaceae). Formosa: Dep. Patiño, Pozo del Tigre, 24-II-1951, Ragonese \& Castiglioni 7946 (LP). 34. Tabernaemontana catharinensis A. DC. (Apocynaceae). Misiones: Dep. Eldorado, Eldorado, 21-III-2005, Martínez 51 (LPAG 3181); Dep. Iguazú, Puerto Iguazú, 18-IX1970, Dimitri \& Amorin 89 (LPAG). Chaco: Dep. Primero de Mayo, Colonia Benítez, X-1931, Schulz 441 (LP). 35. Terminalia triflora (Griseb.) Lillo (Combretaceae). Chaco: Dep. San Fernando, Isla Soto en selva de inundación, 28-XI-1978, Schinini 16142 (LP). Chaco: Dep. San Fernando, Resistencia, 15-IX-1956, Pedersen 3981 (LP). Corrientes: Dep. San Cosme, Paso de la Patria, 30-IX-1987, Krapovickas \& Schinini 41912 (LP). 36. Trichilia catigua A. Juss. (Meliaceae). Corrientes: Dep. Concepción, Concepción, 23-III-1969, Pedersen 9083 (LP 4195). Misiones: Dep. San Javier, Cerro del Monje, 7-IX-1993, Arbo \& al. 5913 (BA 77844). 